\title{
The German Job Search Panel
}

Clemens Hetschko ${ }^{\dagger, *}, *$

Mario Lawes
Julia Schmidtke

Ronnie Schöb:**
Michael Eid

Gesine Stephan ${ }^{ \pm, *}$

This version: 30 December 2022

\begin{abstract}
This report introduces the German Job Search Panel, a monthly survey that follows people who registered as job seeking over the course of up to two years. The focus of the survey is on the well-being and health of jobseekers, with special emphasis on workers affected by mass layoffs. The use of an innovative survey app allows for frequent measurement every month and for conducting the experience sampling method to measure affective well-being. The collected data may be linked to administrative records of the Federal Employment Agency subject to participant consent. For a subsample of surveyed jobseekers hair cortisol levels are available as a biomarker for chronic stress. In this report, we describe the sampling procedure, adjustments over the recruitment period and the collected data. We moreover examine selective participation in the panel. It turns out that high-skilled workers, young individuals and women were more likely to sign up. Age increases the probability to take part in the hair sampling. People working in East Germany are more likely to consent to the linkage of survey data and administrative records. However, the aggregated bias resulting from selective participation is small.
\end{abstract}

\footnotetext{
$\dagger$ University of Leeds

* CESifo, Munich

+ Freie Universität Berlin

\pm Institute for Employment Research (IAB), Nuremberg

$\because:$ Friedrich-Alexanders-Universität Erlangen-Nuremberg

Corresponding author. Clemens Hetschko, Leeds University Business School, Economics Division, Maurice Keyworth Building, Leeds, LS2 9JT.

Acknowledgements. The authors are indebted to the IAB-DIM unit, in particular Stephan Grießemer who carried out the sampling, and thank Benjamin Küfner for valuable research assistance. Financial support by the German Science Foundation (DFG) through projects EI 379/11-1, EI 379/11-2, SCHO 1270/5-1, SCHO 1270/5-2, STE 1424/4-1 and STE 1424/4-2 as well as by the Institute for Employment Research (IAB) through projects 3111, 3874,3877 and 3905 is gratefully acknowledged.
} 


\section{Introduction}

In recent years, well-being indicators have become an important tool for the analysis of the individual impact of unemployment (e.g., Hetschko \& Schöb, 2017; Paul \& Moser, 2009; Wanberg, 2012). However, previous research has mainly focused on one measure of subjective well-being, overall life satisfaction (e.g., Luhmann et al., 2012, 2014; Luhmann \& Eid, 2009), for exceptions, see Knabe et al. (2010)). The German Job Search Panel (GJSP) intends to broaden this perspective. It is a collection of monthly panel data of jobseekers, which allows researchers examining the impact of unemployment on three different dimensions of well-being at the same time - subjective, eudaimonic and physical well-being. In the process, multiple indicators are available for each construct. For instance, subjective well-being can be elicited via different measures of cognitive well-being as well as measures of affective well-being. As a result, the GJSP enables researchers to analyze if the changes in well-being following unemployment vary between well-being constructs. These differences may be mapped over time, with relatively short time lags of one month between the points of measurement.

This data report describes the GJSP. A first focus is on the data generating process. It is based on the notification process of jobseekers with the German Federal Employment Agency (Bundesagentur für Arbeit, BA) and identifies people who consider likely that their employment relationship ends in the course of the following months to invite them to participate in a panel survey about job search. The sampling places special emphasis on mass layoffs as reason for the termination of a job. Participants are then surveyed once a month over a period of up to two years. These comprehensive panel data are collected using an innovative generic smartphone app. The survey data may be linked to high-quality register-based data of the Institute for Employment Research (IAB) and to people's objectively measured chronic stress levels, ascertained by hair cortisol. These two features are further novelties in the research on unemployment and well-being.

During the recruitment of participants, we had to deal with low sign-up rates initially. This issue was aggravated further by the fact that less than half of the persons that signed up could actually be considered for the final sample for methodological reasons. We therefore adjusted the way we contacted participants in several ways over the recruitment period. This included extending the sample to non-mass-layoff-related jobseeker registrations. Thus, a second focus of this data report is to give an account of these adjustments and the evolution of the sign-up rate. Moreover, we describe the scheme used to incentivize repeated participation over time and give an overview of the collected data. 
Unavoidably, sign-up to a smartphone-based survey is non-random. A third focus of this data report is therefore on sample selectivity. This concerns initial participation, survival in the panel over time, consent to linking administrative records to the survey data as well as the willingness to provide sensitive health data through hair cortisol. Our sampling process allows us to investigate selectivity in depth, as we are able to link participation data ('paradata') to the register data available at IAB. While the average non-response bias is only $3.7 \%$ across characteristics, it turns out that females and relatively educated people are significantly more likely to sign up for the study and to continuously participate over many months than males and less educated people. Younger age groups are more likely to participate in general whereas older participants are more willing to provide hair samples for cortisol measurement.

\section{Sampling}

For our data collection, the Data and IT Management (DIM) unit of IAB identified workers who had registered as jobseekers in the German unemployment insurance system prior to entering unemployment. In Germany, to be eligible for insurance-based unemployment benefits (Arbeitslosengeld), employees are obliged to register as job seeking with the employment agency at the latest three months before they expect (having) to terminate their job. If they learn about the termination of their employment later, they will have to register as a jobseeker within three days. Otherwise, a cut-off period for the receipt of unemployment benefits may apply. By contacting them early on when they had just registered as jobseekers, we ensured that our survey would include one measurement wave before workers potentially enter unemployment.

Many people who register as jobseekers, however, do not enter unemployment later on. For instance, if employers announce mass layoffs, it is often unclear for some time who actually loses work and who is able to stay with the firm. Fixed-term contracts are also often prolonged shortly before the expiration date which means that people register as jobseekers pro forma. Hence, many workers who have registered as jobseekers three months before possibly terminating their jobs eventually stay employed at the same firm. Stephan (2016) shows that only slightly more than half of the people that register as job seeking actually become unemployed within the following three months. A relatively high share of registered jobseekers stays employed with the same firm or immediately takes up a new job. The GJSP exploits this institutional background to provide a comparison group of employed workers for individuals entering unemployment. Both groups are surveyed after the registration of jobseekers, whether 
or not they actually become unemployed later on. The fact that both groups start the panel as registered jobseekers ensures some principle similarity.

IAB-DIM obtains the address data of recently registered jobseekers through the data warehouse of the Federal Employment Agency, with a delay of about two to eight weeks after registration. For the period from November 2017 to May 2019, they identified jobseekers that registered between the $15^{\text {th }}$ day of the second-last month to the $14^{\text {th }}$ day of the last month as potential participants of the GJSP. This means we sampled jobseekers during a period of low overall unemployment in Germany, varying between 3\% and $4 \%$ over our sampling phase (OECD, 2022). People were contacted if they met the following conditions ${ }^{1}$ : German nationality (as our surveys were conducted in German language only), age between 18 and 60 years, the current employment relationship is subject to social insurance contributions (sozialversicherungspflichtige Beschäftigung) and has lasted for at least 150 days (i.e., probation is most likely over). Moreover, their registration as jobseeker had not been directly preceded by a previous registration. Partly due to these restrictions, we primarily sampled jobseekers that were eligible for unemployment benefits in case of job loss. ${ }^{2}$

Due to our particular interest in the effects of involuntary unemployment, we started off drawing samples of registered jobseekers who were likely affected by a mass layoff only (over the whole recruitment period, $N=79,092$ invites were sent out to this group). ${ }^{3}$ However, we decided to draw an additional sample from August 2018 onwards as our mass layoff sample had filled up only slowly over time. Therefore, we additionally identified an equally sized random sample of recently registered jobseekers out of the group of people who did not fulfill the requirements of a mass layoff (from August 2018 to May 2019, $N=48,109$ ). While a focus on mass layoffs limits the extent of unobservable heterogeneity, comparing multiple reasons for job search with respect to their impact on well-being is of interest for researchers in itself (Chadi \& Hetschko, 2018).

\footnotetext{
1 IAB-DIM initiated the dispatch of invitation letters and emails through the postal office of the Federal Employment Agency. This procedure also ensured that we did not have access to the address data.

${ }^{2}$ Provided that the unemployed person has been employed for 12 months in the last 30 months, unemployment benefits replace $60 \%$ (people without children) / $67 \%$ (people with children) of the previous net salary. The receipt usually expires after 12 months. The duration is shorter for people who were employed for less than two years before entering unemployment and longer for workers who are at least 50 years old and were employed for at least 30 months before entering unemployment. Afterwards, people are eligible for means-tested welfare benefits (Arbeitslosengeld II).

${ }^{3}$ We apply the thresholds for a notifiable mass layoff according to §17(1) of the German employment protection act (Kündigungsschutzgesetz): > 5 layoffs in plants with 21 to 59 employees, $10 \%$ in plants with 60 to 250 employees, $>25$ layoffs in plants with 251 to 499 employees, $\geq 30$ layoffs in plants with 500 or more employees. In addition, we assume a mass layoff in a plant of $\leq 20$ employees if the staff reduced by more than five people.
} 
As it takes some time to learn about newly registered jobseekers through the data warehouse of the BA, many people had already stopped working when they received the invitation to the study. We therefore used the first couple of questions of the app-based survey ('entry survey') to carry out further exclusions. Most importantly, people who reported to have become unemployed already at the time of the entry survey were not considered further for the study $(N=1,424) \cdot{ }^{4}$ Again, this was to ensure the availability of a pre-event measure of well-being. 215 subjects were excluded because they were still in their probation period. Of the remaining group, every third person was randomly excluded to run an experiment on the effect of participating in the study on future labor market outcomes $(N=940)$. Moreover, 246 individuals did not submit their entry survey. While 4,698 persons signed up for the study initially, only $1,873(40 \%)$ people were kept in the final sample. ${ }^{5}$

The survey was conducted by an independent research institute, the Happiness Research Organization (HRO). The HRO developed a generic app specifically for this purpose, the Happiness Analyzer (Ludwigs \& Erdtmann, 2019). The app was adjusted and used for commercial use such as employee surveys and for research (for previous applications, see, e.g., Hendriks et al., 2016; Ludwigs et al., 2020). ${ }^{6}$ Note that people did not need to be online when participating. Questionnaires and the gathered data were locally stored and only transmitted once people had Wi-Fi access.

\section{Contact modes and adjustments over time}

As shown in Figure 1, the number of identified jobseekers varied substantially across the recruitment period. ${ }^{7}$ Note that the number of people that were invited would be half as high from August 2018 onwards had we not extended invitations to a second sample of non-masslayoff jobseekers. The number of contacted jobseekers peaked in November 2018. This might reflect a high number of people terminating seasonal jobs or having contracts that expire at the end of the year, thus noticing the employment agency in fall.

\footnotetext{
445 individuals claimed to be employed and were included in the final sample even though they were already no longer employed at the time of their jobseeker registration according to later updates of the administrative data.

5 The numbers presented here might slightly differ from substantive analyses based on the GJSP due to specific strategies of dealing with a small number of people who were invited more than once or who potentially falsely claimed to be still employed at sign-up. These cases can be identified via variables in the dataset.

${ }^{6}$ During our recruitment period, over $95 \%$ of smartphones used in Germany relied on Android or iOS as operating systems (Statista, 2022). The Happiness Analyzer runs on both, circumventing selective participation dependent on system software.

7 The figure starts with January 2018 as we only contacted a subsample of the actual target group as a pilot study in November 2017 and December 2017 (1,430 invitees combined).
} 
People were initially contacted via a physical letter sent to their home addresses (Appendix B, Material B1). The letter highlighted the study purposes and informed about the institutions involved. It also provided extensive information on data privacy (Appendix B, Material B2) and included a personal token needed to register for the study. The initial sign-up rate in January 2018 and February 2018 was relatively low (see, again, Figure 1). Less than 2\% of contacted people installed the app and inserted their token successfully. Accordingly, the number of people actually recruited (final sample) was tiny. We therefore decided to augment the letter by an elaborate and professionally designed flyer (see Appendix B, Material B3). Apart from other nicely presented information about the study, it henceforth contained QR codes directing subjects to the Google PlayStore or Apple Appstore to download the survey app, which were formerly part of the letter.

The sign-up rate soared significantly after the flyer was included in the envelope, providing descriptive support for its effectiveness in recruiting people for the study. We kept providing flyers with the physical letters until the end of the recruitment period. Still, the final sample filled up slowly. As mentioned before, we therefore decided to randomly draw an equally sized second sample of non-mass-layoff jobseekers from the eligible group of individuals, doubling the number of potential participants ('dual sampling').

Moreover, if an email address was available to the BA, people were invited via email instead of a physical letter from August 2018 onwards (see Appendix B, Material B4). Emails conveniently enable participants to use a link to download the app on their smartphone. Links can lead to informative websites. However, email invitations may be misinterpreted as spam and fail to convey the legitimacy of the study. It is therefore a priori unclear whether this step increases participation. At least, contacting people by email helps to save postage costs.

The left-hand panel (A) of Figure 2 presents the sign-up rates by mode of contact from August 2018 to October 2018. It turns out that many more people who were contacted by email installed the app and registered for the survey. This does not need to be an effect of the contact mode though. People who provide an email address might differ also in other respects from those who do not, for instance, when it comes to age, openness to using digital tools, concerns about data privacy and thus be more likely to participate in an app-based study than the latter.

To explore these possibilities and to exploit the potential of the GJSP for methodological research, we ran an experiment on the mode of contact from November 2018 until the end of the recruitment period. We randomly assigned three contact modes to equally sized subgroups of those people who provided an email address (see Lawes et al., 2022a): physical letter, email and a physical pre-announcement letter followed by an email (Appendix B, Material B5). 
Figure 1. Contacted jobseekers and sign-up rates over time

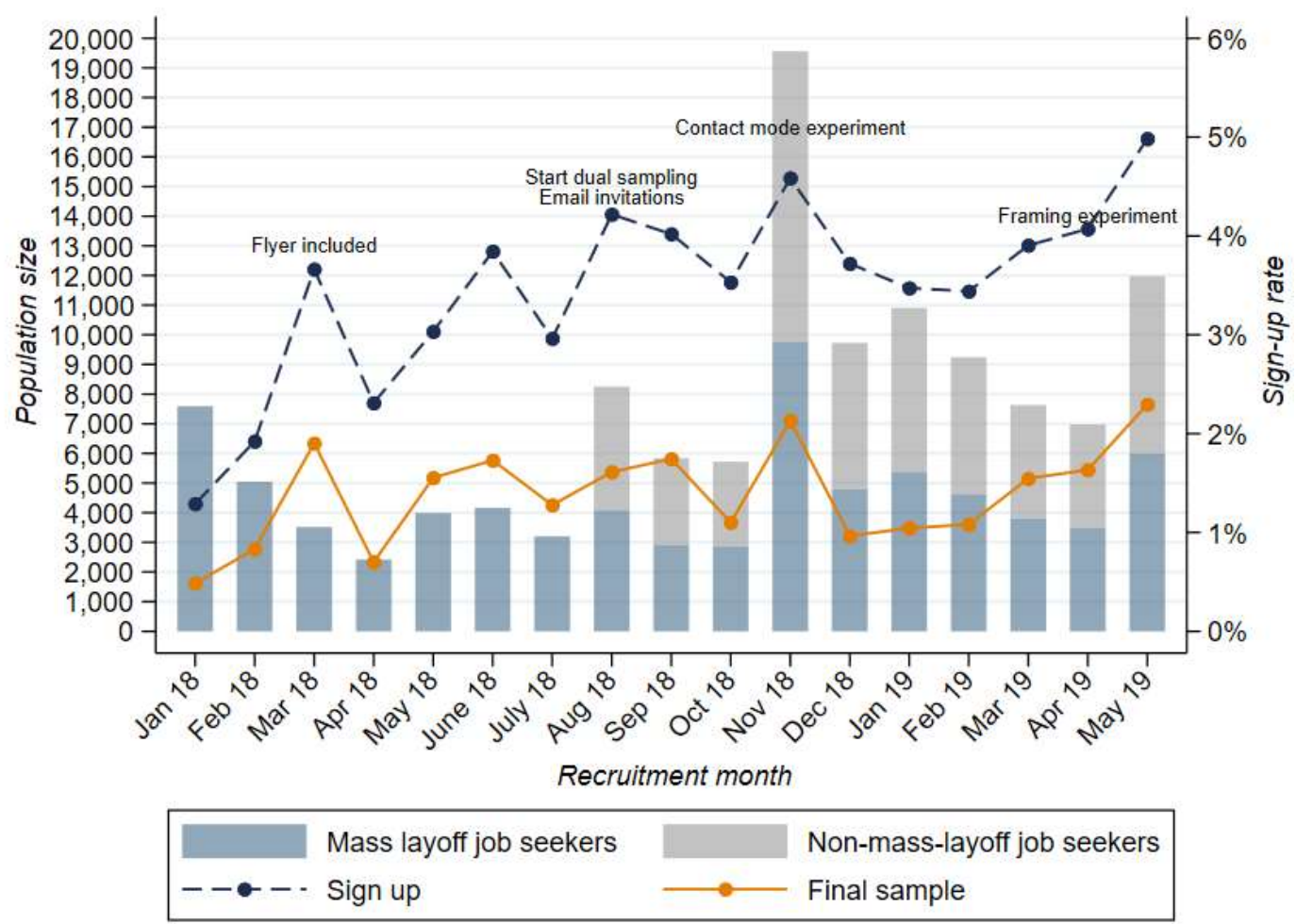

Source. Paradata on GJSP participation.

The results of the experiment are presented in the right-hand panel (B) of Figure 2. Neither physical letters nor emails yield higher participation rates. People that provide an email address are simply also more likely to participate in this kind of study. Sending a pre-announcement letter notifying people that they will receive an email inviting them to the GJSP leads to a significantly higher sign-up rate than the other conditions. It seems to combine the advantages of both modes, conveying the legitimacy of the study (physical pre-announcement letter) and convenience when it comes to downloading the app (follow-up email).

As a final adjustment toward the end of the recruitment period, we focused on individuals who received the letter because they had not provided an email address to the Federal Employment Agency. In particular, we tried to formulate the invitation in a simpler and more appealing way. In addition, we experimented with goal framing, placing stronger emphasis on either the monetary incentives provided by the study or the normative goal of making a contribution to society by participating in a research project (Lindenberg \& Steg, 2007). Against the background of a limited budget and improving sign-up rates, we terminated recruiting further participants in May 2019.

Despite the positive trend in sign-up to the GJSP over time, the participation rate seems relatively low, overall. People might have hesitated to sign up for an obviously burdensome 
study that requires them to indicate plenty of information over a long time every month. Others may have anticipated that they are not part of the target group anymore as they had already lost their jobs. Moreover, people could contact the research team for queries and to provide feedback via telephone and email after they received the invitation. Based on their many calls and messages, we suspect that the extensive public discussion in the run-up to the introduction of the EU's General Data Protection Regulation (GDPR) in spring 2018 did not help in recruiting participants, despite the fact that our study complied with all rules of that directive from the beginning. Other major concerns were of a technical nature, such as uncertainty about one's eligibility for the study, disabled tokens and switching smartphones during participation. ${ }^{8}$

Participation in app-based surveys is generally higher if subjects are recruited from a population of active survey participants, which are obviously prone to take part in studies. Even in these cases the sign-up rate is often below $20 \%$, sometimes only $5 \% .{ }^{9}$ There is a lack of evidence on sign-up rates to app-based panel surveys for samples of people who were not previous survey participants, as in our case. We suspect that people's concerns about data protection with respect to smartphone usage generally limit participation in app-based surveys.

Figure 2. Sign-up rates by mode of contact

A

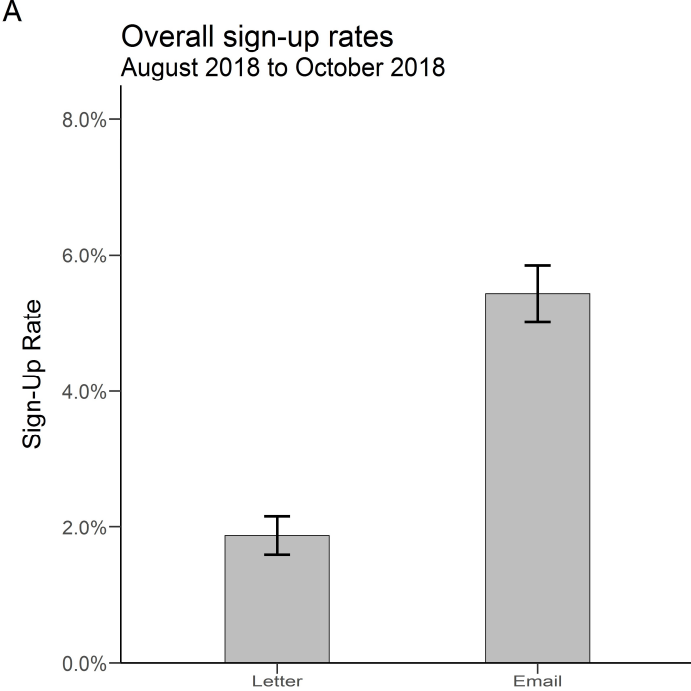

B

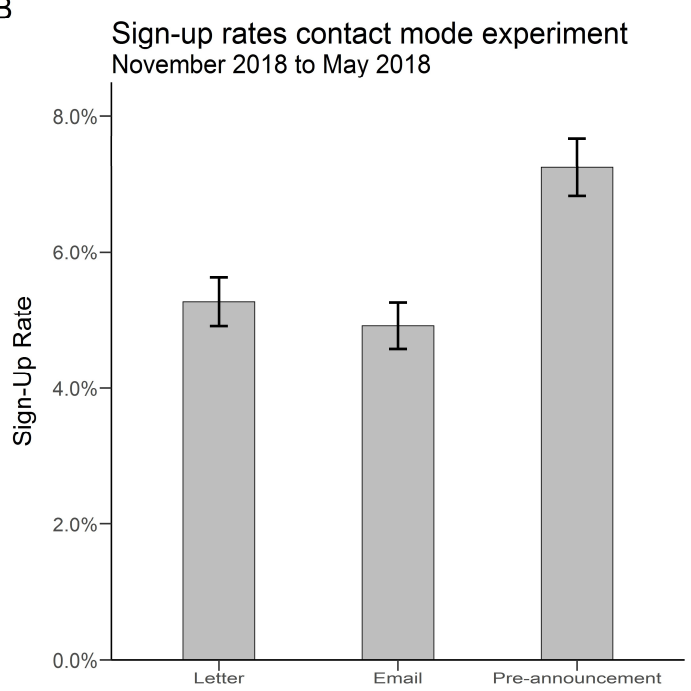

Note. Panel A depicts sign-up rates of people who received a physical letter, as they had not provided an email address and people for which an email address was available. Panel B depicts sign-up rates for people who provided an email address and were randomly assigned to one of the three contact modes. It is based on Lawes et al. (2022a). Whiskers denote 95\% confidence intervals. Source. Paradata on GJSP participation.

\footnotetext{
${ }^{8}$ Once used, tokens needed to be reactivated to avoid multiple participation of the same person.

${ }^{9}$ Kreuter et al. (2019) invited active participants of the German PASS panel to take part in a mobile data collection. Further examples include Scherpenzeel (2017) and Jäckle et al. (2019) based on British UKHLS data. Keusch et al. (2019) invited previously interviewed refugees to provide passive data through an app.
} 


\section{Incentives and smartphone provision}

Continued participation is key to any panel survey. We thus rewarded people who had steadily participated in the study (see Table A1 for an overview). Subjects received 10 euros in the form of a money transfer or an Amazon voucher for each month they completed at least $80 \%$ of all items in the first year. Continued participation was rewarded further by a loyalty bonus of 40 euros after the sixth and the twelfth month. ${ }^{10}$ People who did not reach the threshold of $80 \%$ in two months lost eligibility for the loyalty bonus. Those who sent in hair bundles for the measurement of cortisol got another incentive of 10 euros per hair sample. Self-administered hair sampling entailed extracting a hair bundle and sending it to us in a readymade envelop. Subjects could get feedback on their cortisol values if they completed all five measurements.

Regular incentives were no longer paid during the second year of participation, as the first year was most important for our study purposes. However, people could get lottery tickets in year two. After two years, we drew lots for cash prices worth 5,000 euros in total. Subjects who still participated after April 2020 received a cash incentive of 10 euros per month again because we wanted to ensure that they continued to participate in order to map their well-being around waves of Covid-19. We also continued hair sampling for this group.

An obvious concern about an app-based study is the participation of people who lack access to smartphones. Using apps as survey mode favors the participation of young and educated people as well as people living in areas with widespread broadband internet access. To encourage participation of jobseekers who did not possess a smartphone to participate in the study, we allowed them to complete the entry survey online by using any computer with internet access and to request a smartphone provided through the HRO. Once they received the smartphone, they had to use the app to continue participating. As we will describe in more detail in the next section, the most important reason for us to require smartphones as survey mode is the collection of data on affective well-being via experience sampling. The 179 individuals who borrowed a smartphone did not receive any payments or Amazon vouchers. They could keep the smartphone as a gift after repeatedly participating in the panel study upon the condition that they had completed at least $80 \%$ of all questionnaires in year one. This created an incentive to continue participation over time also for this group of people. The monetary value of the smartphone resembled the money obtained through incentives when participating continuously using one's own device.

\footnotetext{
${ }^{10}$ Initially we paid 15 euros every other month of the first year, ten euros every other month of the second year and no loyalty bonus. The incentive scheme changed for current and new participants.
} 


\section{Collected data}

Once people had successfully installed the survey app, completed the entry survey and were included in the final sample, the app notified them about new questionnaires on up to eight consecutive days each month over a period of two years. This allowed us to balance the burden of participation over several days during a month. The time needed to complete a daily survey was roughly five minutes. As some questions were asked only every three months or six months, participation in months $1,4,7,10,13,16,19,22$ and 25 was more time consuming.

Table 1 details the collected data and the corresponding frequency of measurement. In response to the Covid-19 pandemic, we added items in relation to the event, such as experience with a Covid-19 infection and taking part in the German job retention scheme ('Kurzarbeit, i.e. 'short-time work') as well as a few other items (highlighted bold). The wording of all questions can be found in our codebook on the Open Science Framework's (OSF) webpage of the German Job Search Panel (https://osf.io/h4f25/), unless access to a scale is legally restricted. The collection of well-being data followed guidelines by the OECD and the US National Academy of Sciences (National Research Council, 2013; OECD, 2013). Cognitive well-being (module C) was assessed by the Satisfaction With Life Scale (Diener et al., 1985), Cantril's (1965) ladder and life domain-specific satisfaction indicators.

For measuring affective well-being (module A), we used the experience sampling method (ESM; Hektner et al., 2007; Larson \& Csikszentmihalyi, 1983), among others. Measuring affective well-being is ideally based on real-time information, since retrospective measurement is prone to errors (OECD, 2013). Before the smartphone era, researchers had to equip subjects with pagers that needed to be carried at all times. Now, participants only need to install the app on their smartphone and wait for the ESM notifications, as most of them carry their smartphone anyway. This renders collecting ESM data much less expensive and burdensome for participants than before, allowing for large sample sizes and repeated measurement (Bryson \& MacKerron, 2017).

In our study, people were requested to complete an ESM for their current activity on six randomly chosen time points over the course of one day per month. Those who completed less than three episodes were asked to repeat the ESM module at the end of the monthly questionnaire cycle. The mood states elicited were based on the Multidimensional Mood State Questionnaire (Multidimensionaler Befindlichkeitsfragebogen, see Steyer et al. (1997)). Specifically, panelists were asked about their momentary happiness/unhappiness, awakeness/sleepiness and calmness/restlessness. 
We also employed the day reconstruction method (DRM; Kahneman et al., 2004) to assess affective well-being. The DRM requires people to complete a diary of the previous day with all activities, for which they are then to indicate their emotional state. We ascertained the emotions happy, nervous, sad, worried, enthusiastic, bored, lonely and stressed. Later on, we also included a summary affective well-being measure concerning the previous day. In addition, people completed a German short version of the Center of Epidemiological Studies Depression Scale (CES-D / Allgemeine Depressionsskala, see, e.g., Hautzinger et al. (2012) over the previous week which can be seen as a measure of mental health as well as affective well-being.

Eudaimonic well-being (module E) was measured in three ways. First, by using an adapted 24-item short version of the Ryff(1989) Scale of Psychological Well-Being which was obtained from applying confirmatory factor analysis in combination with an ant algorithm (Schultze, 2017). Four items each assessed the six facets self-acceptance, positive relations, autonomy, environmental mastery, personal growth and purpose in life. Second, by asking individuals during each ESM episode whether their current activity is perceived as meaningful. Third, respondents were asked in the DRM if they had perceived the activity as meaningful.

Further main psychological variables (module P) included a 21-item version of the five facets proactive coping, reflective coping, strategic planning, instrumental support seeking and avoidance coping of the Proactive Coping Inventory (Greenglass et al., 1999). It was reduced to 21 items by a principal component analysis. We also included selected dimensions of the AVEM (Arbeitsbezogenes Verhaltens- und Erlebensmuster, work-related behavior and experience patterns, see Schaarschmidt \& Fischer (2008), a mood regulation inventory (Lischetzke et al., 2001) and the subscale 'perceived available support' of the Berlin Social Support Scales (Schulz \& Schwarzer, 2003). Finally, we augmented our version of the CES-D with similarly worded questions on perceived stress and loneliness.

For the measurement of physical health (module H), we closely followed the German Ageing Survey (DEAS; Engstler et al., 2013) and asked people, inter alia, about their current health status, diagnosed diseases and health expectations. As mentioned before, we also measured hair cortisol as a biomarker for chronic stress (Stalder \& Kirschbaum, 2012). Chronic stress increases the risk for cardiovascular diseases (Dimsdale, 2008), type 2 diabetes (Pouwer et al., 2010), ulcerative colitis (Mawdsley et al., 2006) and decreases fertility (Ebbesen et al., 2009). We further asked subjects to indicate which hair styling products they use, how long they are exposed to the sunlight and whether they use any cortisol-based medication (Dettenborn et al., 2012). The Dresden Lab Service determined the chronic stress level over the past three months in $3 \mathrm{~cm}$ hair segments. 
Table 1. Questionnaire modules

\begin{tabular}{|c|c|c|c|}
\hline \multicolumn{3}{|c|}{ Module } & \multirow{2}{*}{$\begin{array}{c}\text { Frequency } \\
\text { Monthly }\end{array}$} \\
\hline $\mathbf{C}$ & $\mathrm{C} 0$ & Satisfaction With Life Scale & \\
\hline & $\mathrm{C} 1$ & Cantril's ladder & Quarterly \\
\hline & $\mathrm{C} 2$ & Satisfaction with various life domains & Monthly \\
\hline \multirow[t]{4}{*}{$\mathbf{A}$} & A0 & Day reconstruction method (feelings) & Quarterly \\
\hline & A1 & Yesterday's mood (new) & Monthly \\
\hline & $\mathrm{A} 2$ & Experience sampling: Multidimensional Mood State Questionnaire & Monthly \\
\hline & $\mathrm{A} 3$ & Center for Epidemiologic Studies Depression Scale & Monthly \\
\hline \multirow[t]{2}{*}{$\mathbf{E}$} & E0 & Ryff Scale of Psychological Well-Being & Monthly \\
\hline & E1 & Experienced meaningfulness (as part of A0 / A1) & Monthly / quarterly \\
\hline \multirow[t]{7}{*}{$\mathbf{P}$} & $\mathrm{P} 0$ & Proactive Coping Inventory & Monthly \\
\hline & $\mathrm{P} 1$ & Perceived stress (as part of A0 and add-on to A3) & Monthly \\
\hline & $\mathbf{P 2}$ & Loneliness (as part of $\mathrm{A0}$ and later also added to $\mathrm{A3}$ & Monthly \\
\hline & P3 & Work-related Behavior and Experience Patterns (selected dimensions) & Entry survey \\
\hline & $\mathrm{P} 4$ & Tendency to resign and problem coping & Monthly \\
\hline & P5 & Mood regulation questionnaire & Monthly \\
\hline & P6 & Berlin Social Support Scale, Perceived support & Monthly (adjusted) \\
\hline \multirow[t]{14}{*}{$\mathbf{H}$} & HO & Self-assessed health & Monthly \\
\hline & $\mathrm{H} 1$ & Changes in health (last five years) & Entry survey \\
\hline & $\mathrm{H} 2$ & Expected changes in health (next three years) & Entry survey \\
\hline & H3 & Weight & Monthly \\
\hline & $\mathrm{H} 4$ & Disability & Biyearly \\
\hline & H5 & Diseases & Monthly \\
\hline & H6 & Doctoral visits (last three months) & Quarterly \\
\hline & H7 & Overnight stays in hospital (last three months) & Quarterly \\
\hline & $\mathrm{H} 8$ & Height & Entry survey \\
\hline & H9 & Hair cortisol concentration as a biomarker of chronic stress & Quarterly \\
\hline & H10 & Worries about health and beyond (new) & Monthly \\
\hline & H11 & Covid-19 infection (new) & Monthly \\
\hline & H12 & Covid-19 contact (new) & Monthly \\
\hline & H13 & Covid-19 quarantine (new) & Monthly \\
\hline \multirow[t]{10}{*}{$\mathbf{0}$} & 00 & Consent to record linkage & Month 1,2 or 3 \\
\hline & 01 & Employment is still the same & Entry survey \\
\hline & 02 & Start date of employment & Entry survey \\
\hline & 03 & Reason for job seeker registration & Entry survey \\
\hline & 04 & Working hours & Entry survey \\
\hline & 05 & Long hours (adjusted) & Entry survey \\
\hline & 06 & Earnings & Entry survey \\
\hline & 07 & Job characteristics & Entry survey \\
\hline & 08 & Employment-related expecation & Entry survey \\
\hline & 09 & Smartphone awareness & Entry survey \\
\hline \multirow[t]{12}{*}{1} & 10 & Marital status & Biyearly \\
\hline & 11 & Partnership & Biyearly \\
\hline & 12 & Employment status of partner & Biyearly \\
\hline & 13 & Place of residence in 1989 & Entry survey \\
\hline & 14 & Education / training & Biyearly \\
\hline & 15 & Number of children & Biyearly \\
\hline & 16 & Household income & Monthly \\
\hline & 17.1 & Prejudice toward the unemployed (new) & Monthly \\
\hline & 17.2 & Big five personality traits, risk aversion & Quarterly \\
\hline & $\mathbf{1 7 . 3}$ & Patience (new) & Monthly \\
\hline & 18 & Fairness, locus of control & Quarterly \\
\hline & 19 & Religion & Biyearly \\
\hline \multirow[t]{10}{*}{2} & 20 & Labour market status & Monthly \\
\hline & 21 & Termination of employment relationship (adjusted) & Monthly \\
\hline & 22 & Working hours & Quarterly \\
\hline & 23 & Long hours & Quarterly \\
\hline & 24 & Earnings & Quarterly \\
\hline & 25 & Job characteristics & Quarterly \\
\hline & 26 & Employment-related expecations & Monthly (adjusted) \\
\hline & 27 & Kurzarbeit scheme (short-time work, new) & Monthly \\
\hline & 28 & Key worker (new) & Monthly \\
\hline & 29 & Working from home (new) & Monthly \\
\hline \multirow[t]{10}{*}{3} & 30 & Reemployment planning & Monthly \\
\hline & 31 & New job part-time or fulltime & Monthly \\
\hline & 32 & Employability & Monthly \\
\hline & 33 & Reservation wage & Monthly \\
\hline & 34 & Re-employment expectations & Monthly \\
\hline & 35 & Job search & Monthly \\
\hline & 36 & Employment-related expectations & Monthly \\
\hline & 37 & Unemployment benefits (Arbeitslosengeld) & Monthly \\
\hline & 38 & Unemployment benefits II (Arbeitslosengeld II) & Monthly \\
\hline & 39 & Re-employment intentions (new) & Monthly \\
\hline
\end{tabular}

Note. Bold items were added/adjusted in April/July 2020. For all details on the items see the codebook on the OSF webpage of the German Job Search Panel (https://osf.io/h4f25/). 
What is more, we gathered data on multiple individual characteristics (modules numbered 0-3). These were mostly based on the German Socio-Economic Panel (SOEP, see Wagner et al., 2007), on the DEAS and on the survey 'The Value of Work' (Knabe et al., 2010). Subjects were required to indicate their employment status, as we wanted to know when individuals had entered unemployment or found a new job. We also asked them about their job search (if unemployed), job characteristics (if employed), personality traits, family background and so forth. For people who consented to linking the process data of the Federal Employment Agency, information on previous times of employment, unemployment, benefit receipt, participation in labor market policy schemes, among others, can be merged with the survey data.

\section{Selectivity analysis}

\section{Participation}

The final GJSP sample is not representative of the invited group of jobseekers, as we required people to use a smartphone and as the response rate to our study invitation was low. In general, the degree of non-representativeness (i.e., non-response bias) might be the higher the lower the sign-up rate of particular sub-groups (Groves \& Peytcheva, 2008). To document the degree of non-response bias and identify patterns of selective participation, we merge paradata on study participation with the Integrated Employment Biographies (IEB) of the IAB (v16). These register-based data combine individual-level information on periods of dependent employment (not as a public servant though), unemployment/welfare benefit receipt, job seeking and participation in active labor market programs (see Jacobebbinghaus \& Seth, 2007 for more details on the IEB). A small number of individuals was accidentally contacted more than once (636 individuals). In this report, each individual is included with their first jobseeker registration only. Note that paradata on study participation can be linked to the IEB for all contacted persons through an anonymized person identifier. On the contrary, linking survey data with the IEB requires the explicit consent of participants.

All variables are measured at the time of registering as a jobseeker, i.e., shortly before people received the study invitation. We exclude people who, according to the data, earn unrealistically low wages ( $\leq 5$ euros per day) or wages above the ceiling for social insurance contributions (230 euros per day in 2020 for unemployment insurance in Western German federal states), which are prone to measurement error. In addition to the IEB variables, we include the recruitment month and a variable indicating belonging to the mass layoff sample. Table 2 presents descriptive statistics for the invited group of eligible jobseekers, the sample of 
people who signed up and the final sample of people who fulfilled the inclusion criteria. Differences between people who signed up and people who were finally included may be driven by the inclusion criteria. We treat people who did not sign-up as a reference group to test for statistically significant differences between participants and non-participants. It turns out that participants are significantly younger, more likely female, better educated and earn higher wages than non-participants. They also have less work experience over the past ten years. The federal state where people live as well as the recruitment month are also significantly correlated with the probability of signing up. The sign-up rate in the mass-layoff sample was lower compared to the non-mass-layoff samples, but the inclusion criteria seem to have corrected for this bias by chance. What is more, the differences in characteristics between participants and non-participants are highly similar to the whole sample in the group of jobseekers who were contacted as part of the mass layoff sample (cf. Tables 2 and A2 in the Appendix).

\section{Average non-response bias}

One may also calculate the average absolute non-response bias $\left(Y_{\text {absolute }}\right)$ separately for both definitions of participation (people who signed up initially and people who were finally included). In line with the literature (e.g., Sakshaug et al., 2019), we compute proportions $\left(Y_{k}\right)$ of persons who participate $(S)$ and compare them to the group of contacted jobseekers $(J S)$ across a wide range of categories $(k=1, \ldots, K)$ of variables. The average absolute non-response bias is the sum of all absolute differences between the two samples and the contacted individuals $\left(\left|Y_{k, S}-Y_{k, J S}\right|\right)$ for all categories, divided by the total number of categories:

$$
\text { Average Absolute } \text { Bias }_{S}=\frac{\sum_{k=1}^{K}\left|Y_{k, S}-Y_{k, J S}\right|}{K}
$$

For binary variables only one category is considered (e.g., female). Ratio variables need to be categorized to obtain proportions that can be compared between people who signed up / belong to final sample and the eligible jobseekers. If their distribution is highly skewed (e.g., periods of welfare benefit receipt over the past ten years), we collapse them into a binary variable (any indication / no indication). Other ratio variables (e.g., age) are categorized into quartiles of the sample of contacted jobseekers. Table A3 in the Appendix details the corresponding statistics. The absolute non-response bias amounts to $3.0 \%$ in the group of people who signed up and to $3.7 \%$ in the final sample. Non-response biases are thus rather low. This is also true for jobseekers who were invited to participate in the study after experiencing a mass layoff. The average bias is $3.5 \%$ for group who signed up and $4.2 \%$ for final sample, respectively. 
Table 2. Descriptive statistics of invited sample, responders and final sample

\begin{tabular}{|c|c|c|c|c|c|c|c|c|c|c|c|c|}
\hline & \multicolumn{3}{|c|}{ (1) Invited sample } & \multicolumn{3}{|c|}{ (2) Did not sign up } & \multicolumn{3}{|c|}{ (3) Signed up } & \multicolumn{3}{|c|}{ (4) Final sample } \\
\hline & Mean & (std. dv.) & $\mathrm{N}$ & Mean & (std. dv.) & $\mathrm{N}$ & Mean & (std. dv.) & $\mathrm{N}$ & Mean & (std. dv.) & $\mathrm{N}$ \\
\hline Age $(17-60)$ & 41.25 & $(11.23)$ & 127,201 & 41.35 & $(11.24)$ & 122,503 & $38.50^{* * *}$ & $(10.57)$ & 4,698 & $38.62^{* * *}$ & $(10.26)$ & 1,873 \\
\hline Tenure $(.41-22.1)$ & 3.51 & $(4.81)$ & 123,095 & 3.51 & $(4.83)$ & 118,551 & 3.39 & $(4.24)$ & 4,544 & 3.47 & $(4.23)$ & 1,826 \\
\hline \multicolumn{13}{|c|}{ Employment history over past 10 years (measured in years) } \\
\hline Regular employment $(.21-10)$ & 7.27 & $(2.56)$ & 127,196 & 7.29 & $(2.55)$ & 122,498 & $6.81^{* * *}$ & $(2.67)$ & 4,698 & $6.79^{* * *}$ & $(2.65)$ & 1,873 \\
\hline Unemployment benefit receipt $(0-5.33)$ & 0.48 & $(0.69)$ & 127,196 & 0.49 & $(0.69)$ & 122,498 & $0.34^{* * *}$ & $(0.56)$ & 4,698 & $0.33^{* * *}$ & $(0.55)$ & 1,873 \\
\hline Welfare benefit receipt $(0-10)$ & 0.71 & $(1.71)$ & 127,196 & 0.71 & $(1.72)$ & 122,498 & $0.54^{* * *}$ & $(1.46)$ & 4,698 & $0.47^{* * *}$ & $(1.34)$ & 1,873 \\
\hline Registered as jobseeker $(0-10)$ & 1.66 & $(2.06)$ & 127,196 & 1.67 & $(2.07)$ & 122,498 & $1.34^{* * *}$ & $(1.84)$ & 4,698 & $1.30^{* * *}$ & $(1.75)$ & 1,873 \\
\hline Participation in policy schemes $(0-10)$ & 0.28 & $(0.67)$ & 127,196 & 0.28 & $(0.68)$ & 122,498 & $0.23^{* * *}$ & $(0.61)$ & 4,698 & $0.19^{* * *}$ & $(0.54)$ & 1,873 \\
\hline Female (shares) & 0.40 & $(0.49)$ & 127,198 & 0.40 & $(0.49)$ & 122,500 & $0.50^{\dagger \dagger \dagger}$ & $(0.50)$ & 4,698 & $0.51^{\dagger \dagger \dagger}$ & $(0.50)$ & 1,873 \\
\hline \multicolumn{13}{|l|}{ Highest level of qualification (shares) } \\
\hline Missing & 0.00 & $(0.00)$ & 127,201 & 0.00 & $(0.00)$ & 122,503 & $0.00^{\dagger \dagger \dagger}$ & $(0.00)$ & 4,698 & $0.00^{\dagger \dagger}$ & $(0.00)$ & 1,873 \\
\hline None & 0.05 & $(0.21)$ & 127,201 & 0.05 & $(0.22)$ & 122,503 & $0.02^{\dagger \dagger \dagger}$ & $(0.15)$ & 4,698 & $0.02^{\dagger \dagger \dagger}$ & $(0.12)$ & 1,873 \\
\hline Vocational training & 0.61 & $(0.49)$ & 127,201 & 0.62 & $(0.49)$ & 122,503 & $0.35^{\dagger \dagger \dagger}$ & $(0.48)$ & 4,698 & $0.30^{\dagger \dagger \dagger}$ & $(0.46)$ & 1,873 \\
\hline A-levels & 0.01 & $(0.11)$ & 127,201 & 0.01 & $(0.11)$ & 122,503 & $0.02^{\mathrm{t \dagger \dagger}}$ & $(0.14)$ & 4,698 & $0.01^{\dagger \dagger \dagger}$ & $(0.12)$ & 1,873 \\
\hline A-levels and vocational training & 0.14 & $(0.34)$ & 127,201 & 0.13 & $(0.34)$ & 122,503 & $0.19^{\dagger \dagger \dagger}$ & $(0.39)$ & 4,698 & $0.19^{\dagger \dagger \dagger}$ & $(0.39)$ & 1,873 \\
\hline Tertiary degree & 0.19 & $(0.39)$ & 127,201 & 0.18 & $(0.39)$ & 122,503 & $0.42^{\dagger \dagger \dagger}$ & $(0.49)$ & 4,698 & $0.48^{\dagger \dagger \dagger}$ & $(0.50)$ & 1,873 \\
\hline \multicolumn{13}{|l|}{ Federal state (shares) } \\
\hline Schleswig-Holstein & 0.03 & $(0.18)$ & 127,148 & 0.03 & $(0.18)$ & 122,450 & $0.03^{\dagger \dagger \dagger}$ & $(0.17)$ & 4,698 & $0.03^{\dagger \dagger \dagger}$ & $(0.16)$ & 1,873 \\
\hline Hamburg & 0.02 & $(0.14)$ & 127,148 & 0.02 & $(0.14)$ & 122,450 & $0.03^{\dagger \dagger \dagger}$ & $(0.18)$ & 4,698 & $0.03^{\dagger \dagger \dagger}$ & $(0.18)$ & 1,873 \\
\hline Lower Saxony & 0.11 & $(0.31)$ & 127,148 & 0.11 & $(0.31)$ & 122,450 & $0.09^{\dagger \dagger \dagger}$ & $(0.29)$ & 4,698 & $0.10^{\dagger \dagger \dagger}$ & $(0.31)$ & 1,873 \\
\hline Bremen & 0.01 & $(0.10)$ & 127,148 & 0.01 & $(0.10)$ & 122,450 & $0.01^{\mathrm{ft \dagger}}$ & $(0.10)$ & 4,698 & $0.01^{\mathrm{f \dagger \dagger}}$ & $(0.11)$ & 1,873 \\
\hline North Rhine Westphalia & 0.19 & $(0.39)$ & 127,148 & 0.19 & $(0.39)$ & 122,450 & $0.19^{\dagger \dagger \dagger}$ & $(0.39)$ & 4,698 & $0.18^{\dagger \dagger \dagger}$ & $(0.39)$ & 1,873 \\
\hline Hesse & 0.06 & $(0.24)$ & 127,148 & 0.06 & $(0.24)$ & 122,450 & $0.05^{\dagger \dagger \dagger}$ & $(0.22)$ & 4,698 & $0.06^{\dagger \dagger \dagger}$ & $(0.23)$ & 1,873 \\
\hline Rhineland Palatinate & 0.05 & $(0.21)$ & 127,148 & 0.05 & $(0.21)$ & 122,450 & $0.04^{\dagger \dagger \dagger}$ & $(0.19)$ & 4,698 & $0.03^{\dagger \dagger \dagger}$ & $(0.18)$ & 1,873 \\
\hline Baden Württemberg & 0.09 & $(0.29)$ & 127,148 & 0.09 & $(0.29)$ & 122,450 & $0.10^{\text {tiो }}$ & $(0.31)$ & 4,698 & $0.11^{\dagger \dagger \dagger}$ & $(0.31)$ & 1,873 \\
\hline Bavaria & 0.18 & $(0.38)$ & 127,148 & 0.18 & $(0.38)$ & 122,450 & $0.16^{\dagger \dagger \dagger}$ & $(0.37)$ & 4,698 & $0.14^{\dagger \dagger \dagger}$ & $(0.35)$ & 1,873 \\
\hline Saarland & 0.01 & $(0.12)$ & 127,148 & 0.01 & $(0.12)$ & 122,450 & $0.01^{\dagger \dagger \dagger}$ & $(0.11)$ & 4,698 & $0.01^{\dagger \dagger \dagger}$ & $(0.11)$ & 1,873 \\
\hline Berlin & 0.05 & $(0.21)$ & 127,148 & 0.05 & $(0.21)$ & 122,450 & $0.09^{\dagger \dagger \dagger}$ & $(0.29)$ & 4,698 & $0.09^{\dagger \dagger \dagger}$ & $(0.29)$ & 1,873 \\
\hline Brandenburg & 0.04 & $(0.19)$ & 127,148 & 0.04 & $(0.19)$ & 122,450 & $0.04^{\dagger \dagger \dagger}$ & $(0.18)$ & 4,698 & $0.04^{\mathrm{f \dagger \dagger}}$ & $(0.20)$ & 1,873 \\
\hline Mecklenburg Western Pomerania & 0.02 & $(0.15)$ & 127,148 & 0.02 & $(0.15)$ & 122,450 & $0.02^{\mathrm{ft \dagger}}$ & $(0.15)$ & 4,698 & $0.02^{\dagger \dagger \dagger}$ & $(0.15)$ & 1,873 \\
\hline Saxony & 0.06 & $(0.24)$ & 127,148 & 0.06 & $(0.24)$ & 122,450 & $0.06^{\dagger \dagger \dagger}$ & $(0.24)$ & 4,698 & $0.06^{\dagger \dagger}$ & $(0.24)$ & 1,873 \\
\hline Saxony Anhalt & 0.04 & $(0.20)$ & 127,148 & 0.04 & $(0.21)$ & 122,450 & $0.03^{\dagger \dagger \dagger}$ & $(0.18)$ & 4,698 & $0.03^{\dagger \dagger \dagger}$ & $(0.18)$ & 1,873 \\
\hline Thuringia & 0.04 & $(0.20)$ & 127,148 & 0.04 & $(0.20)$ & 122,450 & $0.03^{\dagger \dagger \dagger}$ & $(0.18)$ & 4,698 & $0.04^{\dagger \dagger \dagger}$ & $(0.18)$ & 1,873 \\
\hline Abroad & 0.00 & $(0.02)$ & 127,148 & 0.00 & $(0.02)$ & 122,450 & $0.00^{\dagger \dagger \dagger}$ & $(0.00)$ & 4,698 & $0.00^{\dagger \dagger \dagger}$ & $(0.00)$ & 1,873 \\
\hline Mass layoff sample (share) & 0.62 & $(0.48)$ & 127,201 & 0.62 & $(0.48)$ & 122,503 & $0.56^{\dagger \dagger \dagger}$ & $(0.50)$ & 4,698 & 0.63 & $(0.48)$ & 1,873 \\
\hline
\end{tabular}

Table to be continued on the next page. 


\section{Table 2 (continued)}

\begin{tabular}{|c|c|c|c|c|c|c|c|c|c|c|c|c|}
\hline & \multicolumn{3}{|c|}{ Invited sample } & \multicolumn{3}{|c|}{ Did not sign up } & \multicolumn{3}{|c|}{ Signed up } & \multicolumn{3}{|c|}{ Final sample } \\
\hline & Mean & (std. dv.) & $\mathrm{N}$ & Mean & (std. dv.) & $\mathrm{N}$ & Mean & (std. dv.) & $\mathrm{N}$ & Mean & (std. dv.) & $\mathrm{N}$ \\
\hline \multicolumn{13}{|l|}{ Recruitment month (shares) } \\
\hline Pre-test (November, December 2017) & 0.01 & $(0.11)$ & 127,201 & 0.01 & $(0.11)$ & 122,503 & $0.00^{\dagger \dagger \dagger}$ & $(0.07)$ & 4,698 & $0.00^{\dagger \dagger \dagger}$ & $(0.05)$ & 1,873 \\
\hline January 2018 & 0.06 & $(0.24)$ & 127,201 & 0.06 & $(0.24)$ & 122,503 & $0.02^{\dagger \dagger}$ & $(0.14)$ & 4,698 & $0.02^{\dagger \dagger}$ & $(0.14)$ & 1,873 \\
\hline February 2018 & 0.04 & $(0.20)$ & 127,201 & 0.04 & $(0.20)$ & 122,503 & $0.02^{\dagger \dagger \dagger}$ & $(0.14)$ & 4,698 & $0.02^{\dagger \dagger \dagger}$ & $(0.15)$ & 1,873 \\
\hline March 2018 & 0.03 & $(0.16)$ & 127,201 & 0.03 & $(0.16)$ & 122,503 & $0.03^{\dagger \dagger \dagger}$ & $(0.16)$ & 4,698 & $0.04^{\dagger \dagger \dagger}$ & $(0.19)$ & 1,873 \\
\hline April 2018 & 0.02 & $(0.14)$ & 127,201 & 0.02 & $(0.14)$ & 122,503 & $0.01^{\dagger \dagger \dagger}$ & $(0.11)$ & 4,698 & $0.01^{\dagger \dagger \dagger}$ & $(0.09)$ & 1,873 \\
\hline May 2018 & 0.03 & $(0.17)$ & 127,201 & 0.03 & $(0.17)$ & 122,503 & $0.03^{\dagger \dagger \dagger}$ & $(0.16)$ & 4,698 & $0.03^{\dagger \dagger \dagger}$ & $(0.18)$ & 1,873 \\
\hline June 2018 & 0.03 & $(0.18)$ & 127,201 & 0.03 & $(0.18)$ & 122,503 & $0.03^{\dagger \dagger \dagger}$ & $(0.18)$ & 4,698 & $0.04^{\dagger \dagger \dagger}$ & $(0.19)$ & 1,873 \\
\hline July 2018 & 0.03 & $(0.16)$ & 127,201 & 0.03 & $(0.16)$ & 122,503 & $0.02^{\dagger \dagger \dagger}$ & $(0.14)$ & 4,698 & $0.02^{\dagger \dagger \dagger}$ & $(0.15)$ & 1,873 \\
\hline August 2018 & 0.06 & $(0.25)$ & 127,201 & 0.06 & $(0.25)$ & 122,503 & $0.07^{\dagger \dagger \dagger}$ & $(0.26)$ & 4,698 & $0.07^{i \dagger \dagger}$ & $(0.26)$ & 1,873 \\
\hline September 2018 & 0.05 & $(0.21)$ & 127,201 & 0.05 & $(0.21)$ & 122,503 & $0.05^{\dagger \dagger \dagger}$ & $(0.22)$ & 4,698 & $0.05^{\dagger \dagger \dagger}$ & $(0.23)$ & 1,873 \\
\hline October 2018 & 0.04 & $(0.21)$ & 127,201 & 0.05 & $(0.21)$ & 122,503 & $0.04^{\dagger \dagger \dagger}$ & $(0.20)$ & 4,698 & $0.03^{\dagger \dagger \dagger}$ & $(0.18)$ & 1,873 \\
\hline November 2018 & 0.15 & $(0.36)$ & 127,201 & 0.15 & $(0.36)$ & 122,503 & $0.19^{\dagger \dagger \dagger}$ & $(0.39)$ & 4,698 & $0.22^{\dagger \dagger \dagger}$ & $(0.42)$ & 1,873 \\
\hline December 2018 & 0.08 & $(0.27)$ & 127,201 & 0.08 & $(0.27)$ & 122,503 & $0.08^{\dagger \dagger \dagger}$ & $(0.27)$ & 4,698 & $0.05^{\dagger \dagger \dagger}$ & $(0.22)$ & 1,873 \\
\hline January 2019 & 0.09 & $(0.28)$ & 127,201 & 0.09 & $(0.28)$ & 122,503 & $0.08^{\dagger \dagger \dagger}$ & $(0.27)$ & 4,698 & $0.06^{\dagger \dagger \dagger}$ & $(0.24)$ & 1,873 \\
\hline February 2019 & 0.07 & $(0.26)$ & 127,201 & 0.07 & $(0.26)$ & 122,503 & $0.07^{\dagger \dagger \dagger}$ & $(0.25)$ & 4,698 & $0.05^{\dagger \dagger \dagger}$ & $(0.22)$ & 1,873 \\
\hline March 2019 & 0.06 & $(0.24)$ & 127,201 & 0.06 & $(0.24)$ & 122,503 & $0.06^{\dagger \dagger \dagger}$ & $(0.24)$ & 4,698 & $0.06^{\dagger \dagger \dagger}$ & $(0.24)$ & 1,873 \\
\hline April 2019 & 0.05 & $(0.23)$ & 127,201 & 0.05 & $(0.23)$ & 122,503 & $0.06^{\dagger \dagger \dagger}$ & $(0.24)$ & 4,698 & $0.06^{\dagger \dagger \dagger}$ & $(0.24)$ & 1,873 \\
\hline May 2019 & 0.09 & $(0.29)$ & 127,201 & 0.09 & $(0.29)$ & 122,503 & $0.13^{\dagger \dagger \dagger}$ & $(0.33)$ & 4,698 & $0.15^{\dagger \dagger \dagger}$ & $(0.35)$ & 1,873 \\
\hline \multicolumn{13}{|l|}{ Employment status (shares) } \\
\hline Employed, contributing to social insurance & 0.97 & $(0.18)$ & 127,201 & 0.97 & $(0.18)$ & 122,503 & 0.97 & $(0.18)$ & 4,698 & 0.97 & $(0.16)$ & 1,873 \\
\hline Marginally employed & 0.00 & $(0.04)$ & 127,201 & 0.00 & $(0.04)$ & 122,503 & 0.00 & $(0.04)$ & 4,698 & 0.00 & $(0.03)$ & 1,873 \\
\hline Other (i.e. apprenticeship) & 0.03 & $(0.17)$ & 127,201 & 0.03 & $(0.17)$ & 122,503 & 0.03 & $(0.17)$ & 4,698 & 0.02 & $(0.15)$ & 1,873 \\
\hline Part-time job (share) & 0.24 & $(0.43)$ & 123,095 & 0.23 & $(0.42)$ & 118,551 & $0.29^{\dagger \dagger \dagger}$ & $(0.46)$ & 4,544 & $0.33^{\dagger \dagger \dagger}$ & $(0.47)$ & 1,826 \\
\hline Daily wage $(5.02-229.35)$ & 92.99 & $(42.23)$ & 120,266 & 92.56 & $(41.95)$ & 115,854 & $104.27^{* * *}$ & $(47.73)$ & 4,412 & $108.01^{* * *}$ & $(47.75)$ & 1,795 \\
\hline Individuals & 127,201 & & & 122,503 & & & 4,698 & & & 1,873 & & \\
\hline
\end{tabular}




\section{Multivariate analyses}

Next, we run separate multivariate analyses on the probabilities of signing up and of belonging to the final sample to identify drivers of selective participation. For instance, the finding that people with less work experience in employment subject to social insurance contributions over the last ten years are relatively likely to sign up might be driven by age (see, again, Table 2). Likewise, the fact that both educational attainment and wages increase the probability to participate might originate from one of the two characteristics only. Table 3 presents the corresponding logit estimates and the marginal effects in percentage points of the probability to belong to the respective sample. Still, young individuals, females and part-time workers were relatively likely to sign up and be part of the final sample, despite potential overlap between these groups, which is now controlled for. ${ }^{11}$ A lower daily wage and longer previous spells of receiving unemployment benefits decrease the likelihood of signing up and final sample inclusion. People in the mass layoff sample were less likely to sign up, but more likely to be included in the final sample. As we report in Table A4 in the Appendix, all of the previous findings on significant predictors of participation emerge likewise within the mass layoff sample.

Most strikingly, education has a strong effect on the probability to participate in the GJSP. For example, people with a tertiary degree are about 3.9 percentage points more likely to sign up than people with no degree when all other covariates are at their mean (mass layoff sample: 3.6 p.p.). This stark difference reduces to 1.7 percentage points (mass layoff sample: 1.5 p.p.) when it comes to the probability of belonging to the final sample. Generally, the marginal effects (and, hence, the conditional variable-specific non-response biases) appear smaller for the final sample than for sign up. ${ }^{12}$

\footnotetext{
11 According to Lawes et al. (2022a), the gender difference in sign-up would not exist had we been able to invite everyone by a pre-announcement letter and a subsequent email. Males are almost as likely to sign up as females in the corresponding treatment condition of their experiment.

12 Note that this observation is not based on tests for statistically significant differences.
} 
Table 3. Probability of signing up and belonging to the final sample, conditional estimates

\begin{tabular}{|c|c|c|c|c|c|c|}
\hline & Prol & bility of sign & $n g u p$ & Probabi & $\begin{array}{l}\text { y of belongi } \\
\text { sample }\end{array}$ & $g$ to final \\
\hline & $\begin{array}{c}\text { Logit } \\
\text { estimates }\end{array}$ & $\begin{array}{c}\text { (Standard } \\
\text { errors) }\end{array}$ & $\begin{array}{c}\text { Marginal } \\
\text { effects }\end{array}$ & $\begin{array}{c}\text { Logit } \\
\text { estimates }\end{array}$ & $\begin{array}{c}\text { (Standard } \\
\text { errors) }\end{array}$ & $\begin{array}{c}\text { Marginal } \\
\text { effects }\end{array}$ \\
\hline Age in years / 10 & $-0.181^{* * *}$ & $(0.018)$ & $-0.507^{* * *}$ & $-0.187^{* * *}$ & $(0.028)$ & $-0.194^{* * *}$ \\
\hline Tenure in years / 10 & 0.031 & $(0.044)$ & 0.086 & 0.058 & $(0.068)$ & 0.060 \\
\hline Employment history (over past 10 years) & & & & & & \\
\hline Regular employment $(.21-10)$ & -0.011 & $(0.008)$ & -0.030 & -0.022 & $(0.012)$ & -0.023 \\
\hline Unemployment benefit receipt $(0-5.33)$ & $-0.146^{* * *}$ & $(0.041)$ & $-0.409^{* * *}$ & $-0.208^{* * *}$ & $(0.063)$ & $-0.216^{* * *}$ \\
\hline Welfare benefit receipt $(0-10)$ & $-0.060^{*}$ & $(0.024)$ & $-0.167^{*}$ & $-0.165^{* * *}$ & $(0.035)$ & $-0.171^{* * *}$ \\
\hline Registered as jobseeker $(0-10)$ & $0.046^{*}$ & $(0.022)$ & $0.130^{*}$ & $0.139^{* * *}$ & $(0.031)$ & $0.144^{* * *}$ \\
\hline Participation in policy schemes $(0-10)$ & 0.056 & $(0.029)$ & 0.156 & -0.009 & $(0.050)$ & -0.009 \\
\hline Female (shares) & $0.248^{* * *}$ & $(0.034)$ & $0.696^{* * *}$ & $0.282^{* * *}$ & $(0.053)$ & $0.292^{* * *}$ \\
\hline Level of qualification (ref. no degree) & & & & & & \\
\hline Vocational training & $0.339^{* *}$ & $(0.107)$ & $0.950^{* *}$ & $0.479^{*}$ & $(0.193)$ & $0.497^{*}$ \\
\hline A-levels & $1.084^{* * *}$ & $(0.151)$ & $3.039^{* * *}$ & $1.021^{* * *}$ & $(0.271)$ & $1.060^{* * *}$ \\
\hline A-levels and vocational training & $1.067^{* * *}$ & $(0.110)$ & $2.993^{* * *}$ & $1.316^{* * *}$ & $(0.196)$ & $1.366^{* * *}$ \\
\hline Tertiary degree & $1.397^{* * *}$ & $(0.109)$ & $3.917^{* * *}$ & $1.666^{* * *}$ & $(0.194)$ & $1.729^{* * *}$ \\
\hline Federal state (ref. Schleswig Holstein) & & & & & & \\
\hline Hamburg & 0.228 & $(0.126)$ & 0.640 & 0.368 & $(0.201)$ & 0.382 \\
\hline Lower Saxony & -0.024 & $(0.104)$ & -0.068 & 0.234 & $(0.170)$ & 0.243 \\
\hline Bremen & -0.044 & $(0.184)$ & -0.123 & 0.288 & $(0.266)$ & 0.299 \\
\hline North Rhine Westphalia & 0.017 & $(0.098)$ & 0.047 & 0.135 & $(0.163)$ & 0.140 \\
\hline Hesse & -0.217 & $(0.114)$ & -0.608 & -0.000 & $(0.183)$ & -0.000 \\
\hline Rhineland Palatinate & -0.019 & $(0.121)$ & -0.054 & 0.089 & $(0.199)$ & 0.092 \\
\hline Baden Württemberg & 0.062 & $(0.103)$ & 0.175 & 0.215 & $(0.170)$ & 0.223 \\
\hline Bavaria & 0.049 & $(0.099)$ & 0.137 & 0.080 & $(0.166)$ & 0.083 \\
\hline Saarland & -0.005 & $(0.169)$ & -0.013 & 0.097 & $(0.273)$ & 0.100 \\
\hline Berlin & $0.358^{* * *}$ & $(0.106)$ & $1.005^{* * *}$ & $0.416^{*}$ & $(0.174)$ & $0.432^{*}$ \\
\hline Brandenburg & 0.168 & $(0.123)$ & 0.470 & $0.518^{* *}$ & $(0.193)$ & $0.538^{* *}$ \\
\hline Mecklenburg Western Pomerania & 0.153 & $(0.141)$ & 0.430 & $0.455^{*}$ & $(0.219)$ & $0.472^{*}$ \\
\hline Saxony & 0.115 & $(0.110)$ & 0.323 & 0.216 & $(0.181)$ & 0.224 \\
\hline Saxony Anhalt & 0.089 & $(0.125)$ & 0.249 & 0.295 & $(0.202)$ & 0.306 \\
\hline Thuringia & 0.095 & $(0.125)$ & 0.267 & 0.347 & $(0.199)$ & 0.360 \\
\hline Mass layoff sample & $-0.114^{* *}$ & $(0.035)$ & $-0.321^{* *}$ & $0.123^{*}$ & $(0.055)$ & $0.128^{*}$ \\
\hline Recruitment month (ref. November 2018) & & & & & & \\
\hline Pre-test (November, December 2017) & $-0.638^{* *}$ & $(0.220)$ & $-1.788^{* *}$ & $-1.397^{* *}$ & $(0.453)$ & $-1.450^{* *}$ \\
\hline January 2018 & $-0.607^{* * *}$ & $(0.113)$ & $-1.703^{* * *}$ & $-0.790^{* * *}$ & $(0.180)$ & $-0.820^{* * *}$ \\
\hline February 2018 & $-0.296^{* *}$ & $(0.113)$ & $-0.830^{* *}$ & $-0.367^{*}$ & $(0.169)$ & $-0.381^{*}$ \\
\hline March 2018 & 0.030 & $(0.101)$ & 0.083 & 0.019 & $(0.142)$ & 0.020 \\
\hline April 2018 & $-0.336^{*}$ & $(0.144)$ & $-0.943^{*}$ & $-0.786^{* *}$ & $(0.251)$ & $-0.816^{* *}$ \\
\hline May 2018 & $-0.314^{* *}$ & $(0.103)$ & $-0.881^{* *}$ & $-0.347^{*}$ & $(0.143)$ & $-0.360^{*}$ \\
\hline June 2018 & -0.027 & $(0.092)$ & -0.075 & -0.131 & $(0.133)$ & -0.136 \\
\hline July 2018 & $-0.277^{*}$ & $(0.116)$ & $-0.778^{*}$ & $-0.422^{*}$ & $(0.172)$ & $-0.438^{*}$ \\
\hline August 2018 & -0.060 & $(0.068)$ & -0.168 & $-0.203^{*}$ & $(0.103)$ & $-0.210^{*}$ \\
\hline September 2018 & -0.003 & $(0.079)$ & -0.009 & -0.029 & $(0.117)$ & -0.030 \\
\hline October 2018 & -0.057 & $(0.085)$ & -0.159 & $-0.364^{*}$ & $(0.143)$ & $-0.378^{*}$ \\
\hline December 2018 & -0.016 & $(0.067)$ & -0.046 & $-0.508^{* * *}$ & $(0.119)$ & $-0.528^{* * *}$ \\
\hline January 2019 & 0.001 & $(0.066)$ & 0.004 & $-0.334^{* *}$ & $(0.110)$ & $-0.346^{* *}$ \\
\hline February 2019 & -0.087 & $(0.069)$ & -0.245 & $-0.403^{* * *}$ & $(0.114)$ & $-0.419^{* * *}$ \\
\hline March 2019 & 0.018 & $(0.072)$ & 0.050 & -0.046 & $(0.108)$ & -0.047 \\
\hline April 2019 & -0.029 & $(0.074)$ & -0.081 & -0.106 & $(0.113)$ & -0.110 \\
\hline May 2019 & $0.172^{* *}$ & $(0.056)$ & $0.481^{* *}$ & $0.163^{*}$ & $(0.081)$ & $0.169^{*}$ \\
\hline Part-time job (share) & $0.189^{* * *}$ & $(0.040)$ & $0.530^{* * *}$ & $0.401^{* * *}$ & $(0.061)$ & $0.417^{* * *}$ \\
\hline Daily wage in euros / 10 & $0.043^{* * *}$ & $(0.004)$ & $0.120^{* * *}$ & $0.062^{* * *}$ & $(0.007)$ & $0.065^{* * *}$ \\
\hline Individuals & 120,187 & & 120,187 & 117,570 & & 117,570 \\
\hline
\end{tabular}

Note. The table presents logit estimates and marginal effects (at means, percentage points) of the probability to sign up / belong to the final sample of the GJSP survey. The number of individuals is lower compared to Table 2 since only individuals without any missing information in the relevant variables are included in the estimation. ${ }^{* * *} p<0.001,{ }^{* *} p<0.01,{ }^{*} p<0.05$.

Source. Paradata on GJSP participation and IEB version $v 16$. 


\section{Compliance with record linkage and hair sampling}

All analyses considering hair cortisol or linking survey data to administrative data would be subject to further sample selection unless the willingness to provide hair samples or to give consent to the record linkage was random. Table A5 in the Appendix displays to what extent the final sample differs from the groups who expressed this willingness. It also provides figures on another relevant comparison group, the subsample of people that were asked to send hair samples / consent to record linkage which is not the same as the final sample. Differences in this respect may originate from attrition up to the points where we inquired if people were prepared to comply with our requests (cortisol: end of the first week of questions, record linkage: months two and three). However, Table A5 reveals hardly any differences. People who approve of linking their administrative records to their survey data resemble the final sample largely. Hence, in this respect the non-response bias is small.

The high number of compliers (cortisol: $81.8 \%$, linkage consent: $90.7 \%$ and $81.4 \%$ and $90.4 \%$ for the mass layoff sample, respectively) may cover important reasons for belonging to the exceptional group of people that does not give their consent to the record linkage / is unwilling to submit hair samples. We therefore run a multivariate analysis, separately estimating the propensities of consent to record linkage and indicated willingness to participate in the cortisol study based on the respective samples of people who were asked to comply. The results of the corresponding logit estimations are depicted by Table 4.

We find a couple of substantial marginal effects. The effects of only three individual characteristics, however, are statistically significant: age increases the probability of participating in the cortisol study. While we can only speculate about the reason for this finding, it seems plausible that older people are more interested in health-related studies. This is also true for the mass layoff sample as we show in Table A6 (Appendix), where, additionally, the daily wage attracts a statistically significantly negative effect. A much more detailed analysis of participation in the hair sampling can be obtained from Lawes et al. (2022b). In the process, further obstacles to participation after indicating a principal willingness are analyzed and discussed. For instance, not all willing individuals could actually participate in the hairsampling as their hair was too short. What is more, not all eligible subjects stuck to completing the whole process which includes retrieving and sending a sample of their hair by postal letter. 
People working in East Germany have a higher propensity to consent to the linkage of their administrative records in both the full sample as well as the mass layoff subsample. Time spent regularly employed (i.e., employed subject to social security contributions) increases the propensity to indicate a willingness to partake in the cortisol study, too. This result is not statistically significant in the mass layoff sample, where, however, part-time work attracts a significantly positive effect.

Table 4. Determinants of linkage consent and hair sampling participation

\begin{tabular}{|c|c|c|c|c|c|c|}
\hline & \multicolumn{3}{|c|}{ Probability of Linkage consent } & \multicolumn{3}{|c|}{$\begin{array}{l}\text { Probability of willingness to } \\
\text { participate in the hair sampling }\end{array}$} \\
\hline & $\begin{array}{c}\text { Logit } \\
\text { estimates }\end{array}$ & $\begin{array}{l}\text { (Standard } \\
\text { errors) }\end{array}$ & $\begin{array}{c}\text { Marginal } \\
\text { effects }\end{array}$ & $\begin{array}{l}\text { Logit } \\
\text { estimates }\end{array}$ & $\begin{array}{l}\text { (Standard } \\
\text { errors) }\end{array}$ & $\begin{array}{c}\text { Marginal } \\
\text { effects }\end{array}$ \\
\hline Age in years / 10 & 0.233 & $(0.128)$ & 1.688 & $0.276^{* *}$ & $(0.090)$ & $3.923^{* *}$ \\
\hline Tenure in years / 10 & -0.494 & $(0.287)$ & -3.576 & -0.182 & $(0.206)$ & -2.596 \\
\hline \multicolumn{7}{|l|}{ Employment history (over past 10 years) } \\
\hline Regular employment $(.21-10)$ & $0.111^{*}$ & $(0.052)$ & $0.803^{*}$ & 0.023 & $(0.036)$ & 0.326 \\
\hline Unemployment benefit receipt $(0-5.33)$ & -0.524 & $(0.287)$ & -3.792 & 0.009 & $(0.208)$ & 0.129 \\
\hline Welfare benefit receipt $(0-10)$ & -0.110 & $(0.158)$ & -0.794 & 0.102 & $(0.116)$ & 1.452 \\
\hline Registered as jobseeker $(0-10)$ & 0.033 & $(0.144)$ & 0.239 & -0.136 & $(0.101)$ & -1.934 \\
\hline Participation in policy schemes $(0-10)$ & 0.413 & $(0.273)$ & 2.993 & 0.095 & $(0.176)$ & 1.356 \\
\hline Female (shares) & -0.354 & $(0.217)$ & -2.562 & 0.121 & $(0.153)$ & 1.728 \\
\hline Mass layoff sample & -0.247 & $(0.234)$ & -1.786 & -0.082 & $(0.165)$ & -1.168 \\
\hline Academic degree & 0.307 & $(0.249)$ & 2.224 & 0.064 & $(0.175)$ & 0.909 \\
\hline East Germany & $0.972^{* *}$ & $(0.340)$ & $7.036^{* *}$ & 0.257 & $(0.198)$ & 3.656 \\
\hline \multicolumn{7}{|l|}{ Recruitment month (ref. November 2018) } \\
\hline Pre-test (November, December 2017) & 0.000 & (.) & 0.000 & 0.000 & (.) & 0.000 \\
\hline January 2018 & -0.601 & $(0.706)$ & -4.352 & 0.403 & $(0.653)$ & 5.740 \\
\hline February 2018 & 0.728 & $(1.085)$ & 5.273 & 0.049 & $(0.658)$ & 0.700 \\
\hline March 2018 & 0.987 & $(1.061)$ & 7.145 & -0.205 & $(0.496)$ & -2.924 \\
\hline April 2018 & 0.000 & (.) & 0.000 & 0.955 & $(1.056)$ & 13.598 \\
\hline May 2018 & -0.307 & $(0.510)$ & -2.223 & -0.111 & $(0.411)$ & -1.582 \\
\hline June 2018 & 0.634 & $(0.574)$ & 4.589 & 0.058 & $(0.404)$ & 0.831 \\
\hline July 2018 & 0.577 & $(0.782)$ & 4.179 & $-1.007^{*}$ & $(0.435)$ & $-14.332^{*}$ \\
\hline August 2018 & -0.018 & $(0.411)$ & -0.128 & -0.132 & $(0.306)$ & -1.884 \\
\hline September 2018 & 0.724 & $(0.637)$ & 5.245 & -0.380 & $(0.356)$ & -5.416 \\
\hline October 2018 & 1.196 & $(1.047)$ & 8.663 & -0.063 & $(0.481)$ & -0.896 \\
\hline December 2018 & 0.096 & $(0.484)$ & 0.693 & $-0.740^{*}$ & $(0.319)$ & $-10.529^{*}$ \\
\hline January 2019 & 0.254 & $(0.484)$ & 1.840 & -0.503 & $(0.318)$ & -7.156 \\
\hline February 2019 & -0.014 & $(0.430)$ & -0.102 & 0.468 & $(0.389)$ & 6.667 \\
\hline March 2019 & 0.322 & $(0.452)$ & 2.329 & 0.188 & $(0.342)$ & 2.672 \\
\hline April 2019 & 0.387 & $(0.520)$ & 2.803 & 0.155 & $(0.369)$ & 2.201 \\
\hline May 2019 & -0.032 & $(0.317)$ & -0.234 & -0.426 & $(0.232)$ & -6.061 \\
\hline Part-time job (share) & 0.399 & $(0.276)$ & 2.892 & 0.252 & $(0.196)$ & 3.581 \\
\hline Daily wage in euros / 10 & -0.008 & $(0.031)$ & -0.055 & -0.035 & $(0.022)$ & -0.491 \\
\hline Individuals & 1,194 & & 1,194 & 1,341 & & 1,341 \\
\hline
\end{tabular}

Note. The table presents logit estimates and marginal effects (at means, percentage points) of the two outcomes (linkage consent, cortisol participation), conditional on being asked to comply with the respective outcome. ${ }^{* * *} p<0.001,{ }^{* *} p<0.01,{ }^{*} p<0.05$.

Source. Paradata on GJSP participation and IEB version $v 16$. 


\section{Panel stability}

The upper panel of Figure 4 depicts the retention rates for each month of the first year of the study. Both the number of people missing the $80 \%$ threshold as well as the number of people failing to respond to any item in a given months are displayed. Panel attrition seems to slow down over time. In particular, the $80 \%$ threshold was reached by a stable number of participants from the second month onwards. One might speculate whether the first month was used by many participants as a trial.

The lower panel of Figure 4 depicts retention rates across recruitment quarters based on the number of people that answered at least one item. It turns out that panel attrition was high at the beginning of the study. The recruitment waves in the first quarter of 2018 are associated with the worst retention. This might be due to technical difficulties with the app-based survey during this time, which could explain why about $30 \%$ of participants successfully recruited in the first quarter of 2018 failed to answer at least a single item in the first month. As depicted in Figure 4, the following recruitment quarters perform much better in this respect, which suggests that the underlying problem was solved.

To further analyze panel stability over time, we estimate the probabilities to participate in the survey after 6 months (wave 7, M7) and one year (wave 13, M13), respectively, conditional on the characteristics previously used to explain initial sign-up (see Table 3). Table 5 shows to what extent the participants in M7 and M13 differ from the final sample. We find only a small number of significant results. Females are more likely to still participate in wave 7 of the survey than males. In the subsample of mass layoffs this result is still statistically significant at M13 (see, in addition, Table A7 in the Appendix). Individuals with longer tenures (insignificant in mass layoff sample) and A-level education (but no higher degree) are more likely to continue participation at least until month 13. Again, the first monthly recruitment waves are associated with much larger attrition than later waves. Overall, this means that the sample did not become substantially more selective over time. 
Figure 4. Panel stability
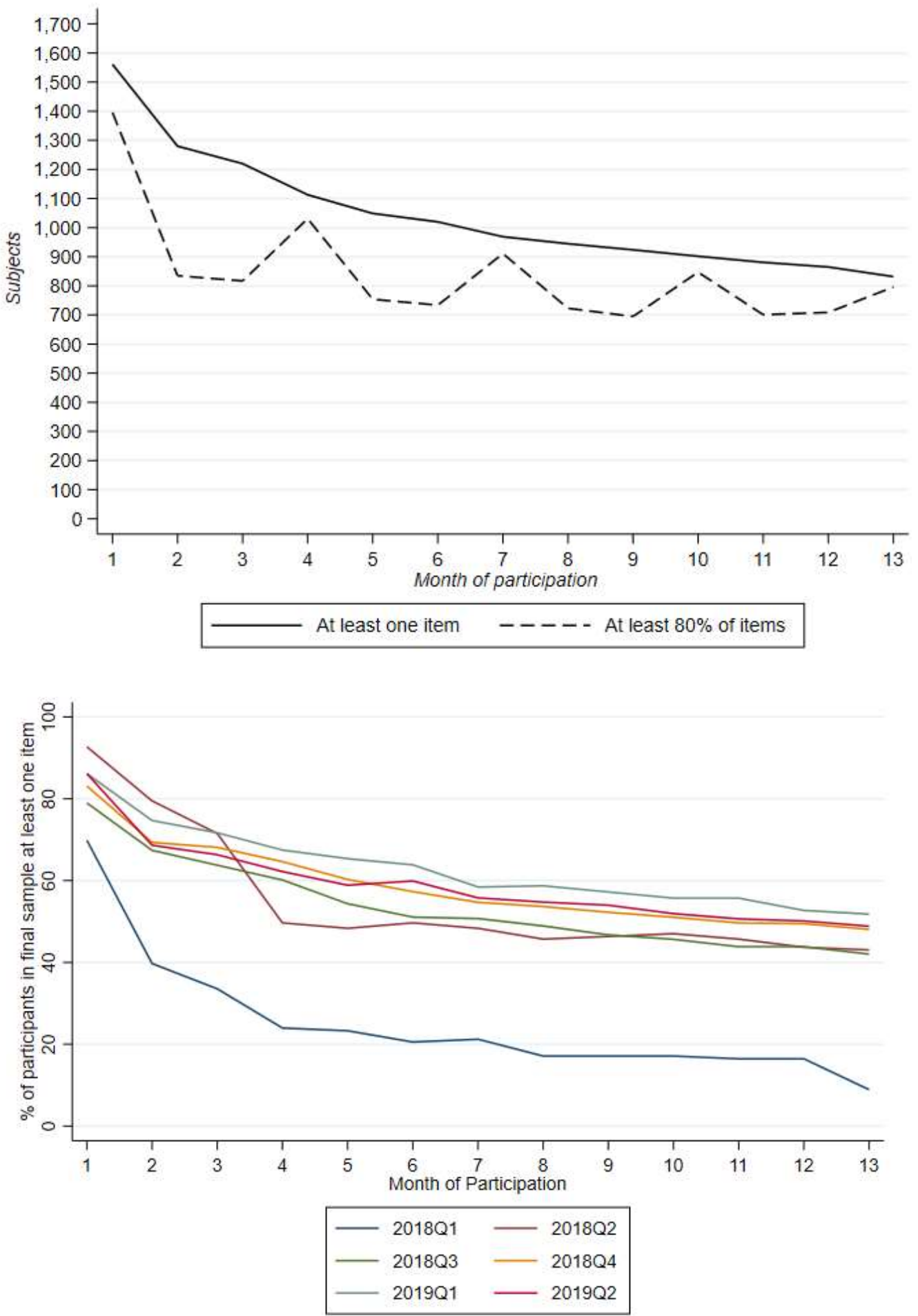

Source. Paradata on GJSP participation.

Note. The solid (dashed) line in the upper panel of the figure depicts the number of participants responding to at least one (80\%) of the questions each month. The lower panel depicts the share of participants included in the final sample responding to at least one item per month for each quarterly period of recruitment. 
Table 5. Determinants of continuous participation of at least six months / one year

\begin{tabular}{|c|c|c|c|c|c|c|}
\hline & \multicolumn{3}{|c|}{ Probability of participating in $M 7$} & \multicolumn{3}{|c|}{ Probability of participating in $M 13$} \\
\hline & $\begin{array}{c}\text { Logit } \\
\text { estimates }\end{array}$ & $\begin{array}{c}\text { (Standard } \\
\text { errors) }\end{array}$ & $\begin{array}{c}\text { Marginal } \\
\text { effects }\end{array}$ & $\begin{array}{c}\text { Logit } \\
\text { estimates }\end{array}$ & $\begin{array}{c}\text { (Standard } \\
\text { errors) }\end{array}$ & $\begin{array}{c}\text { Marginal } \\
\text { effects }\end{array}$ \\
\hline Age in years / 10 & 0.028 & $(0.058)$ & 0.700 & 0.087 & $(0.059)$ & 2.152 \\
\hline Tenure in years / 10 & 0.258 & $(0.145)$ & 6.447 & $0.294^{*}$ & $(0.147)$ & $7.309^{*}$ \\
\hline \multicolumn{7}{|l|}{ Employment history (over past 10 years) } \\
\hline Regular employment $(0.21-10)$ & 0.012 & $(0.025)$ & 0.294 & 0.001 & $(0.025)$ & 0.034 \\
\hline Unemployment benefit receipt $(0-5.33)$ & -0.135 & $(0.139)$ & -3.364 & -0.129 & $(0.142)$ & -3.195 \\
\hline Welfare benefit receipt $(0-10)$ & -0.088 & $(0.077)$ & -2.198 & -0.146 & $(0.079)$ & -3.627 \\
\hline Registered as jobseeker $(0-10)$ & 0.078 & $(0.069)$ & 1.941 & 0.091 & $(0.070)$ & 2.250 \\
\hline Participation in policy schemes $(0-10)$ & -0.115 & $(0.110)$ & -2.882 & -0.056 & $(0.114)$ & -1.382 \\
\hline Female (shares) & $0.209^{*}$ & $(0.106)$ & $5.215^{*}$ & 0.143 & $(0.107)$ & 3.559 \\
\hline \multicolumn{7}{|l|}{ Level of qualification (ref. no degree) } \\
\hline Vocational training & 0.186 & $(0.434)$ & 4.637 & 0.422 & $(0.484)$ & 10.471 \\
\hline A-levels & 0.836 & $(0.583)$ & 20.898 & $1.263^{*}$ & $(0.628)$ & $31.353^{*}$ \\
\hline A-levels and vocational training & 0.499 & $(0.439)$ & 12.466 & 0.674 & $(0.488)$ & 16.719 \\
\hline Tertiary degree & 0.555 & $(0.436)$ & 13.875 & 0.737 & $(0.485)$ & 18.301 \\
\hline \multicolumn{7}{|l|}{ Federal state (ref. Schleswig Holstein) } \\
\hline Hamburg & -0.685 & $(0.418)$ & -17.120 & -0.585 & $(0.423)$ & -14.521 \\
\hline Lower Saxony & -0.240 & $(0.358)$ & -5.991 & -0.042 & $(0.359)$ & -1.043 \\
\hline Bremen & -0.700 & $(0.561)$ & -17.478 & -0.568 & $(0.591)$ & -14.112 \\
\hline North Rhine Westphalia & -0.530 & $(0.344)$ & -13.229 & -0.319 & $(0.345)$ & -7.929 \\
\hline Hesse & -0.382 & $(0.385)$ & -9.544 & -0.216 & $(0.385)$ & -5.370 \\
\hline Rhineland Palatinate & -0.306 & $(0.416)$ & -7.635 & -0.183 & $(0.418)$ & -4.548 \\
\hline Baden Württemberg & -0.513 & $(0.357)$ & -12.813 & -0.297 & $(0.358)$ & -7.368 \\
\hline Bavaria & $-0.713^{*}$ & $(0.349)$ & $-17.823^{*}$ & -0.484 & $(0.351)$ & -12.017 \\
\hline Saarland & 0.364 & $(0.585)$ & 9.093 & -0.197 & $(0.576)$ & -4.883 \\
\hline Berlin & -0.512 & $(0.364)$ & -12.795 & -0.256 & $(0.365)$ & -6.364 \\
\hline Brandenburg & -0.150 & $(0.405)$ & -3.756 & 0.018 & $(0.407)$ & 0.454 \\
\hline Mecklenburg Western Pomerania & 0.076 & $(0.466)$ & 1.896 & 0.072 & $(0.472)$ & 1.775 \\
\hline Saxony & -0.387 & $(0.382)$ & -9.670 & -0.230 & $(0.383)$ & -5.715 \\
\hline Saxony Anhalt & -0.624 & $(0.426)$ & -15.578 & -0.489 & $(0.436)$ & -12.145 \\
\hline Thuringia & -0.457 & $(0.416)$ & -11.418 & -0.315 & $(0.418)$ & -7.810 \\
\hline Mass layoff sample & 0.090 & $(0.112)$ & 2.243 & 0.125 & $(0.111)$ & 3.099 \\
\hline \multicolumn{7}{|l|}{ Recruitment month (ref. November 2018) } \\
\hline Pre-test (November, December 2017) & 0.000 & (.) & 0.000 & 0.000 & (.) & 0.000 \\
\hline January 2018 & $-1.604^{* * *}$ & $(0.477)$ & $-40.063^{* * *}$ & 0.000 & (.) & 0.000 \\
\hline February 2018 & $-1.366^{* * *}$ & $(0.403)$ & $-34.120^{* * *}$ & 0.000 & (.) & 0.000 \\
\hline March 2018 & $-1.385^{* * *}$ & $(0.327)$ & $-34.593^{* * *}$ & $-1.413^{* * *}$ & $(0.348)$ & $-35.086^{* * *}$ \\
\hline April 2018 & -0.590 & $(0.514)$ & -14.746 & -0.568 & $(0.527)$ & -14.094 \\
\hline May 2018 & -0.241 & $(0.290)$ & -6.020 & -0.197 & $(0.291)$ & -4.883 \\
\hline June 2018 & -0.310 & $(0.268)$ & -7.733 & -0.327 & $(0.271)$ & -8.123 \\
\hline July 2018 & -0.015 & $(0.348)$ & -0.370 & -0.020 & $(0.348)$ & -0.507 \\
\hline August 2018 & -0.121 & $(0.208)$ & -3.021 & -0.343 & $(0.211)$ & -8.520 \\
\hline September 2018 & -0.048 & $(0.238)$ & -1.211 & -0.144 & $(0.240)$ & -3.570 \\
\hline October 2018 & -0.087 & $(0.289)$ & -2.169 & -0.293 & $(0.294)$ & -7.269 \\
\hline December 2018 & 0.032 & $(0.241)$ & 0.792 & -0.050 & $(0.242)$ & -1.234 \\
\hline January 2019 & -0.098 & $(0.222)$ & -2.460 & -0.001 & $(0.222)$ & -0.015 \\
\hline February 2019 & $0.660^{* *}$ & $(0.243)$ & $16.478^{* *}$ & $0.477^{*}$ & $(0.233)$ & $11.837^{*}$ \\
\hline March 2019 & 0.228 & $(0.220)$ & 5.696 & 0.114 & $(0.217)$ & 2.818 \\
\hline April 2019 & 0.019 & $(0.228)$ & 0.471 & -0.052 & $(0.229)$ & -1.283 \\
\hline May 2019 & 0.070 & $(0.165)$ & 1.747 & 0.057 & $(0.164)$ & 1.418 \\
\hline Part-time job (share) & 0.110 & $(0.129)$ & 2.739 & 0.044 & $(0.130)$ & 1.092 \\
\hline Daily wage in euros / 10 & -0.002 & $(0.015)$ & -0.038 & -0.003 & $(0.015)$ & -0.076 \\
\hline Individuals & 1,790 & & 1,790 & 1,713 & & 1,713 \\
\hline
\end{tabular}

Note. The table presents logit estimates and marginal effects (at means, percentage points) of continued participation after six months and after one year, respectively (conditional on belonging to the final sample). The number of individuals is lower compared to the number of individuals in Table 2 since only individuals without any missing values on the relevant variables are included in the estimations.

${ }^{* *} p<0.001,{ }^{* *} p<0.01,{ }^{*} p<0.05$.

Source. Paradata on GJSP participation and IEB version $v 16$. 


\section{Conclusions}

The GJSP is a new source of panel data with a focus on well-being and health. Jobseekers are surveyed on a monthly basis using a smartphone app. The data collection is innovative along several dimensions: It allows for collecting longitudinal data on various well-being facets and chronic stress using state-of-the-art methods (e.g., experience sampling and hair cortisol) before and after a job loss. Further, the survey data may be linked to rich administrative records covering participants full employment history with a few exceptions (e.g. periods as public servant). The sampling allows for comparing unemployed jobseekers to a highly comparable group of continuously employed individuals. These merits, however, come at some cost, first, low participation, and, second, selective participation.

The low sign-up proved to be an issue, bearing the risk of large non-response bias. Reassuringly, the aggregate bias turned out to be quite low. Nevertheless, the question arises as to why people hesitate to sign up for an app-based survey. The act of downloading, registering, and actively engaging with an app for frequent measurement may still be burdensome. Moreover, people might be concerned about the protection of their data in particular if smartphones are used as survey mode. Not surprisingly, an app-based survey on well-being and health attracts a non-random group of participants. Although aggregate bias is low, there are patterns of self-selection into the sample, most importantly of women, highly educated people and young workers.

On a more general note, when choosing a survey mode, low sign-up and selective participation need to be considered and weighed against the advantages of using smartphone apps over traditional web surveys or face-to-face and telephone surveys. These advantages include convenient high-frequency assessment, increased flexibility for participants, push notifications, reminders and interventions. The case of the GJSP highlights one of these advantages. Data such as experience sampling would be difficult or almost impossible to gather by other means than via a smartphone app. 


\section{References}

Bryson, A., \& MacKerron, G. (2017). Are you happy while you work? Economic Journal, 127(599), 106-125. https://doi.org/10.1111/ecoj.12269

Cantril, H. (1965). Pattern of human concerns. Rutgers University Press.

Chadi, A., \& Hetschko, C. (2018). The magic of the new: How job changes affect job satisfaction. Journal of Economics and Management Strategy, 27(1), 23-39. https://doi.org/10.1111/jems.12217

Dettenborn, L., Tietze, A., Kirschbaum, C., \& Stalder, T. (2012). The assessment of cortisol in human hair: Associations with sociodemographic variables and potential confounders. Stress, 15(6), 578-588. https://doi.org/10.3109/10253890.2012.654479

Diener, E., Emmons, R. A., Larsen, R. J., \& Griffin, S. (1985). The Satisfaction With Life Scale. Journal of Personality Assessment, 49(1), 71-75. https://doi.org/10.1207/s15327752jpa4901_13

Dimsdale, J. E. (2008). Psychological stress and cardiovascular disease. Journal of the American College of Cardiology, 51(13), 1237-1246. https://doi.org/10.1016/j.jacc.2007.12.024

Ebbesen, S. M. S., Zachariae, R., Mehlsen, M. Y., Thomsen, D., Højgaard, A., Ottosen, L., Petersen, T., \& Ingerslev, H. J. (2009). Stressful life events are associated with a poor in-vitro fertilization (IVF) outcome: A prospective study. Human Reproduction, 24(9), 2173-2182. https://doi.org/10.1093/humrep/dep185

Engstler, H., Schmiade, N., \& Lejeune, C. (2013). Deutscher Alterssurvey (DEAS ): Kurzbeschreibung des Datensatzes SUF DEAS2011, Version 1.0. 49(Juni), 1-42. https://doi.org/10.5156/DEAS.2011.D.001

Greenglass, E., Schwarzer, R., Jakubiec, D., Fiksenbaum, L., \& Taubert, S. (1999). The Proactive Coping Inventory (PCI): A Multidimensional research instrument. 20th International Conference of the Stress and Anxiety Research Society (STAR), Cracow, Poland. https://estherg.info.yorku.ca/files/2014/09/pci.pdf 
Hautzinger, M., Bailer, M., Hofmeister, D., \& Keller, F. (2012). Allgemeine Depressions Skala. Manual. 2. Überarbeitete und neu normierte Auflage. Göttingen: Hofgrefe Verlag GmbH \& Co KG.

Hektner, J. M., Schmidt, J. A., \& Csikszentmihalyi, M. (2007). Experience sampling method: Measuring the quality of everyday life. Sage Publications.

Hendriks, M., Ludwigs, K., \& Veenhoven, R. (2016). Why are locals happier than internal migrants? The role of daily life. Social Indicators Research, 125(2), 481-508. https://doi.org/10.1007/s11205-014-0856-7

Hetschko, C., \& Schöb, R. (2017). Modes of employment and identity. Die Unternehmung, 71(4), 368-389. https://doi.org/10.5771/0042-059x-2017-4-368

Jäckle, A., Burton, J., Couper, M. P., \& Lessof, C. (2019). Participation in a mobile app survey to collect expenditure data as part of a large-scale probability household panel: Coverage and participation rates and biases. Survey Research Methods, 13(1), 23-44. https://doi.org/10.18148/srm/2019.v13i1.7297

Jacobebbinghaus, P., \& Seth, S. (2007). The German Integrated Employment Biographies Sample IEBS. Schmollers Jahrbuch, 127, 335-342.

Kahneman, D., Krueger, A. B., Schkade, D. A., Schwarz, N., \& Stone, A. A. (2004). A survey method for characterizing daily life experience: The day reconstruction method. Science (New York, N.Y.), 306(5702), 1776-1780. https://doi.org/10.1126/science.1103572

Keusch, F., Struminskaya, B., Antoun, C., Couper, M. P., \& Kreuter, F. (2019). Willingness to participate in passive mobile data collection. Public Opinion Quarterly, 83(S1), 210-235. https://doi.org/10.1093/poq/nfz007

Knabe, A., Rätzel, S., Schöb, R., \& Weimann, J. (2010). Dissatisfied with life but having a good day: Time-use and well-being of the unemployed. Economic Journal, 120(547), 867-889. https://doi.org/10.1111/j.1468-0297.2009.02347.x

Kreuter, F., Haas, G. C., Keusch, F., Bähr, S., \& Trappmann, M. (2019). Collecting survey and smartphone sensor data with an app: Opportunities and challenges around privacy 
and informed consent. Social Science Computer Review, 1-17. https://doi.org/10.1177/0894439318816389

Larson, R., \& Csikszentmihalyi, M. (1983). The experience sampling method. In New directions for methodology of social and behavioral science (Vol. 15, Issue 15, pp. 41-56). https://doi.org/10.1177/0894439305281844

Lawes, M., Hetschko, C., Sakshaug, J. W., \& Grießemer, S. (2022a). Contact modes and participation in app-based smartphone surveys: Evidence from a large-scale experiment. Social Science Computer Review, 40(5), 1076-1092. https://doi.org/10.1177/0894439321993832

Lawes, M., Hetschko, C., Sakshaug, J. W., \& Eid, M. (2022b). Hair cortisol collection in app-based smartphone panel surveys: Feasibility, selective participation, and effects on panel attrition [unpublished manuscript]. https://osf.io/mt2q4/?view_only=7cadc1a648e34a638d3301762f01d451

Lindenberg, S., \& Steg, L. (2007). Normative, gain and hedonic goal frames guiding environmental behavior. Journal of Social Issues, 63(1), 117-137.

Lischetzke, T., Eid, M., Wittig, F., \& Trierweiler, L. (2001). Die Wahrnehmung eigener und fremder Gefühle. Konstruktion und Validierung von Skalen zur Erfassung der emotionalen Selbst-und Fremdaufmerksamkeit sowie der Klarheit über Gefühle. Diagnostica.

Ludwigs, K., \& Erdtmann, S. (2019). The Happiness Analyzer - developing a new technique for measuring subjective well-being. International Journal of Community Well-Being, l(2), 101-114. https://doi.org/10.1007/s42413-018-0011-3

Ludwigs, K., Lucas, R., Veenhoven, R., Richter, D., \& Arends, L. (2020). Can happiness apps generate nationally representative datasets? - A case study collecting data on people's happiness using the german socio-economic panel. Applied Research in Quality of Life, 15(4), 1135-1149. https://doi.org/10.1007/s11482-019-09723-2

Luhmann, M., \& Eid, M. (2009). Does it really feel the same? Changes in life satisfaction following repeated life events. Journal of Personality and Social Psychology, 97(2), 363-381. https://doi.org/10.1037/a0015809 
Luhmann, M., Hofmann, W., Eid, M., \& Lucas, R. E. (2012). Subjective well-being and adaptation to life events: A meta-analysis. Journal of Personality and Social Psychology, 102(3), 592-615. https://doi.org/10.1037/a0025948

Luhmann, M., Weiss, P., Hosoya, G., \& Eid, M. (2014). Honey, I got fired! A longitudinal dyadic analysis of the effect of unemployment on life satisfaction in couples. Journal of Personality and Social Psychology, 107(1), 163-180.

https://doi.org/10.1037/a0036394

Mawdsley, J. E., Macey, M. G., Feakins, R. M., Langmead, L., \& Rampton, D. S. (2006). The effect of acute psychologic stress on systemic and rectal mucosal measures of inflammation in ulcerative colitis. Gastroenterology, 131(2), 410-419. https://doi.org/10.1053/j.gastro.2006.05.017

National Research Council. (2013). Subjective well-being: Measuring happiness, suffering, and other dimensions of experience (A. Stone \& C. Mackie, Eds.). The National Academies Press.

OECD. (2013). OECD guidelines on measuring subjective well-being. OECD Publishing. https://doi.org/10.1787/9789264191655-en

OECD. (2022). Employment: Unemployment rate, by sex and age group. https://stats.oecd.org/index.aspx?queryid=54743

Paul, K. I., \& Moser, K. (2009). Unemployment impairs mental health: Meta-analyses. Journal of Vocational Behavior, 74(3), 264-282. https://doi.org/10.1016/j.jvb.2009.01.001

Pouwer, F., Kupper, N., \& Adriaanse, M. C. (2010). Does emotional stress cause type 2 diabetes mellitus? A review from the European Depression in Diabetes (EDID) Research Consortium. Discovery Medicine, 9(45), 112-118.

Ryff, C. D. (1989). Happiness is everything, or is it? Explorations on the meaning of psychological well-being. Journal of Personality and Social Psychology, 57(6), 10691081. https://doi.org/10.1037/0022-3514.57.6.1069 
Sakshaug, J. W., Vicari, B., \& Couper, M. P. (2019). Paper, e-mail, or both? Effects of contact mode on participation in a web survey of establishments. Social Science Computer Review, 37(6), 750-765. https://doi.org/10.1177/0894439318805160

Schaarschmidt, U., \& Fischer, A. (2008). Arbeitsbezogenes Verhaltens- und Erlebensmuster (AVEM). Manual.

Scherpenzeel, A. (2017). Mixing online panel data collection with innovative methods. In S. Eifler \& F. Faulbaum (Eds.), Methodische Probleme von Mixed-Mode-Ansätzen in der Umfrageforschung (pp. 27-49). Springer.

Schultze, M. (2017). Constructing subtests using ant colony optimization.

Schulz, U., \& Schwarzer, R. (2003). Soziale Unterstützung bei der Krankheitsbewältigung: Die Berliner Social Support Skalen (BSSS). Diagnostica. https://doi.org/10.1026//0012-1924.49.2.73

Stalder, T., \& Kirschbaum, C. (2012). Analysis of cortisol in hair-State of the art and future directions. Brain, Behavior, and Immunity, 26(7), 1019-1029. https://doi.org/10.1016/j.bbi.2012.02.002

Statista (2022). Marktanteile der führenden mobilen Betriebssysteme an der Internetnutzung mit Mobiltelefonen in Deutschland von Januar 2009 bis September 2022 [Data set]. https://de.statista.com/statistik/daten/studie/184332/umfrage/marktanteil-der-mobilenbetriebssysteme-in-deutschland-seit-2009/

Stephan, G. (2016). Arbeitsuchend, aber (noch) nicht arbeitslos: Was kommt nach der Meldung? WSI Mitteilungen, 292-299.

Steyer, R., Schwenkmezger, P., Notz, P., \& Eid, M. (1997). Der Mehrdimensionale Befindlichkeitsfragebogen (MDBF) [The Multidimensional Mood State Questionnaire $(M D M Q)]$. Hogrefe Verlag.

Wagner, G. G., Frick, J. R., \& Schupp, J. (2007). The German Socio-Economic Panel Study (SOEP) - Scope, evolution and enhancements. Schmollers Jahrbuch, 127, 139-169. Wanberg, C. R. (2012). The individual experience of unemployment. Annual Review of Psychology, 63(1), 369-396. https://doi.org/10.1146/annurev-psych-120710-100500 
Appendix A: supplementary tables

Table A1. Incentive scheme

\begin{tabular}{|c|c|c|c|c|c|}
\hline Month & $\begin{array}{l}\text { Monthly } \\
\text { bonus }\end{array}$ & $\begin{array}{l}\text { Loyalty } \\
\text { bonus }\end{array}$ & $\begin{array}{l}\text { Incentive per } \\
\text { month }\end{array}$ & $\begin{array}{c}\text { Sum of } \\
\text { incentives }\end{array}$ & $\begin{array}{c}\text { Sum of } \\
\text { lottery tickets }\end{array}$ \\
\hline 1 & $10.00 €$ & & $10.00 €$ & $10.00 €$ & \\
\hline 2 & $10.00 €$ & & $10.00 €$ & $20.00 €$ & \\
\hline 3 & $10.00 €$ & & $10.00 €$ & $30.00 €$ & \\
\hline 4 & $10.00 €$ & & $10.00 €$ & $40.00 €$ & \\
\hline 5 & $10.00 €$ & & $10.00 €$ & $50.00 €$ & \\
\hline 6 & $10.00 €$ & $40.00 €$ & $50.00 €$ & $100.00 €$ & \\
\hline 7 & $10.00 €$ & & $10.00 €$ & $110.00 €$ & \\
\hline 8 & $10.00 €$ & & $10.00 €$ & $120.00 €$ & \\
\hline 9 & $10.00 €$ & & $10.00 €$ & $130.00 €$ & \\
\hline 10 & $10.00 €$ & & $10.00 €$ & $140.00 €$ & \\
\hline 11 & $10.00 €$ & & $10.00 €$ & $150.00 €$ & \\
\hline 12 & $10.00 €$ & $40.00 €$ & $50.00 €$ & $200.00 €$ & \\
\hline 13 & & & & $200.00 €$ & 2 \\
\hline 14 & & & & $200.00 €$ & 4 \\
\hline 15 & & & & $200.00 €$ & 6 \\
\hline 16 & & & & $200.00 €$ & 8 \\
\hline 17 & & & & $200.00 €$ & 10 \\
\hline 18 & & & & $200.00 €$ & 12 \\
\hline 19 & & & & $200.00 €$ & 14 \\
\hline 20 & & & & $200.00 €$ & 16 \\
\hline 21 & & & & $200.00 €$ & 18 \\
\hline 22 & & & & $200.00 €$ & 20 \\
\hline 23 & & & & $200.00 €$ & 22 \\
\hline 24 & & & & $200.00 €$ & 24 \\
\hline 25 & & & & $200.00 €$ & 26 \\
\hline
\end{tabular}


Table A2. Descriptive statistics mass layoff samples

\begin{tabular}{|c|c|c|c|c|c|c|c|c|c|c|c|c|}
\hline & \multicolumn{3}{|c|}{ (1) Invited sample } & \multicolumn{3}{|c|}{ (2) Did not sign up } & \multicolumn{3}{|c|}{ (3) Signed up } & \multicolumn{3}{|c|}{ (4) Final sample } \\
\hline & Mean & (std. dv.) & $\mathrm{N}$ & Mean & (std. dv.) & $\mathrm{N}$ & Mean & (std. dv.) & $\mathrm{N}$ & Mean & (std. dv.) & $\mathrm{N}$ \\
\hline Age $(17-60)$ & 41.99 & $(11.06)$ & 79,092 & 42.10 & $(11.07)$ & 76,474 & $38.62^{* * *}$ & $(10.42)$ & 2,618 & $38.48^{* * *}$ & $(10.13)$ & 1,174 \\
\hline Tenure $(0.41-22.1)$ & 3.84 & $(5.14)$ & 77,903 & 3.84 & $(5.16)$ & 75,336 & 3.69 & $(4.44)$ & 2,567 & 3.73 & $(4.36)$ & 1,153 \\
\hline \multicolumn{13}{|c|}{ Employment history over past 10 years (measured in years) } \\
\hline Regular employment $(0-10)$ & 7.52 & $(2.50)$ & 79,089 & 7.54 & $(2.49)$ & 76,471 & $6.90^{* * *}$ & $(2.71)$ & 2,618 & $6.86^{* * *}$ & (2.69) & 1,174 \\
\hline Unemployment benefit receipt $(0-4.93)$ & 0.49 & $(0.71)$ & 79,089 & 0.50 & $(0.71)$ & 76,471 & $0.31^{* * *}$ & $(0.54)$ & 2,618 & $0.30^{* * *}$ & $(0.50)$ & 1,174 \\
\hline Welfare benefit receipt $(0-10)$ & 0.62 & $(1.61)$ & 79,089 & 0.63 & $(1.62)$ & 76,471 & $0.50^{* * *}$ & $(1.41)$ & 2,618 & $0.43^{* * *}$ & $(1.27)$ & 1,174 \\
\hline Registered as job seeker $(0-10)$ & 1.59 & $(2.00)$ & 79,089 & 1.60 & $(2.00)$ & 76,471 & $1.27^{* * *}$ & $(1.81)$ & 2,618 & $1.23^{* * *}$ & $(1.68)$ & 1,174 \\
\hline Participation in policy schemes $(0-10)$ & 0.24 & $(0.63)$ & 79,089 & 0.24 & $(0.63)$ & 76,471 & $0.20^{* * *}$ & $(0.58)$ & 2,618 & $0.16^{* * *}$ & $(0.50)$ & 1,174 \\
\hline Female (shares) & 0.35 & $(0.48)$ & 79,090 & 0.34 & $(0.47)$ & 76,472 & $0.45^{\dagger \dagger \dagger}$ & $(0.50)$ & 2,618 & $0.49^{\dagger \dagger \dagger}$ & $(0.50)$ & 1,174 \\
\hline \multicolumn{13}{|l|}{ Highest level of qualification (shares) } \\
\hline Missing & 0.00 & $(0.00)$ & 79,092 & 0.00 & $(0.00)$ & 76,474 & $0.00^{\dagger \dagger \dagger}$ & $(0.00)$ & 2,618 & $0.00^{\dagger \dagger \dagger}$ & $(0.00)$ & 1,174 \\
\hline None & 0.05 & $(0.22)$ & 79,092 & 0.05 & $(0.22)$ & 76,474 & $0.02^{\dagger \dagger \dagger}$ & $(0.14)$ & 2,618 & $0.02^{\dagger \dagger \dagger}$ & $(0.14)$ & 1,174 \\
\hline Vocational training & 0.63 & $(0.48)$ & 79,092 & 0.64 & $(0.48)$ & 76,474 & $0.35^{\dagger \dagger \dagger}$ & $(0.48)$ & 2,618 & $0.30^{\dagger \dagger \dagger}$ & $(0.46)$ & 1,174 \\
\hline A-levels & 0.01 & $(0.11)$ & 79,092 & 0.01 & $(0.11)$ & 76,474 & $0.02^{\dagger \dagger}$ & $(0.14)$ & 2,618 & $0.02^{\dagger \dagger \dagger}$ & $(0.13)$ & 1,174 \\
\hline A-levels and vocational training & 0.12 & $(0.32)$ & 79,092 & 0.12 & $(0.32)$ & 76,474 & $0.16^{\dagger \dagger \dagger}$ & $(0.37)$ & 2,618 & $0.16^{\dagger \dagger \dagger}$ & $(0.37)$ & 1,174 \\
\hline Tertiary degree & 0.19 & $(0.39)$ & 79,092 & 0.18 & $(0.38)$ & 76,474 & $0.45^{\dagger \dagger}$ & $(0.50)$ & 2,618 & $0.50^{\dagger \dagger \dagger}$ & $(0.50)$ & 1,174 \\
\hline \multicolumn{13}{|l|}{ Federal state (shares) } \\
\hline Schleswig-Holstein & 0.03 & $(0.17)$ & 79,055 & 0.03 & $(0.17)$ & 76,437 & $0.03^{\dagger \dagger \dagger}$ & $(0.16)$ & 2,618 & $0.03^{\dagger \dagger}$ & $(0.16)$ & 1,174 \\
\hline Hamburg & 0.02 & $(0.13)$ & 79,055 & 0.02 & $(0.13)$ & 76,437 & $0.03^{\dagger \dagger \dagger}$ & $(0.17)$ & 2,618 & $0.03^{\dagger \dagger \dagger}$ & $(0.17)$ & 1,174 \\
\hline Lower Saxony & 0.11 & $(0.31)$ & 79,055 & 0.11 & $(0.31)$ & 76,437 & $0.10^{\dagger \dagger \dagger}$ & $(0.30)$ & 2,618 & $0.11^{\dagger \dagger \dagger}$ & $(0.32)$ & 1,174 \\
\hline Bremen & 0.01 & $(0.10)$ & 79,055 & 0.01 & $(0.10)$ & 76,437 & $0.01^{\dagger \dagger}$ & $(0.10)$ & 2,618 & $0.01^{\dagger \dagger \dagger}$ & $(0.12)$ & 1,174 \\
\hline North Rhine Westphalia & 0.18 & $(0.38)$ & 79,055 & 0.18 & $(0.38)$ & 76,437 & $0.18^{\dagger \dagger \dagger}$ & $(0.39)$ & 2,618 & $0.19^{\dagger \dagger \dagger}$ & $(0.39)$ & 1,174 \\
\hline Hesse & 0.06 & $(0.23)$ & 79,055 & 0.06 & $(0.23)$ & 76,437 & $0.05^{\dagger \dagger \dagger}$ & $(0.21)$ & 2,618 & $0.05^{\dagger \dagger \dagger}$ & $(0.21)$ & 1,174 \\
\hline Rhineland Palatinate & 0.04 & $(0.21)$ & 79,055 & 0.04 & $(0.21)$ & 76,437 & $0.03^{\dagger \dagger \dagger}$ & $(0.18)$ & 2,618 & $0.03^{\dagger \dagger \dagger}$ & $(0.17)$ & 1,174 \\
\hline Baden Württemberg & 0.08 & $(0.28)$ & 79,055 & 0.08 & $(0.28)$ & 76,437 & $0.10^{\dagger \dagger \dagger}$ & $(0.30)$ & 2,618 & $0.10^{\dagger \dagger \dagger}$ & $(0.30)$ & 1,174 \\
\hline Bavaria & 0.19 & $(0.40)$ & 79,055 & 0.20 & $(0.40)$ & 76,437 & $0.16^{\dagger \dagger \dagger}$ & $(0.37)$ & 2,618 & $0.14^{\dagger \dagger \dagger}$ & $(0.35)$ & 1,174 \\
\hline Saarland & 0.01 & $(0.12)$ & 79,055 & 0.01 & $(0.12)$ & 76,437 & $0.01^{\dagger \dagger \dagger}$ & $(0.10)$ & 2,618 & $0.01^{\dagger \dagger \dagger}$ & $(0.09)$ & 1,174 \\
\hline Berlin & 0.05 & $(0.21)$ & 79,055 & 0.05 & $(0.21)$ & 76,437 & $0.10^{\dagger \dagger \dagger}$ & $(0.30)$ & 2,618 & $0.10^{\dagger \dagger \dagger}$ & $(0.30)$ & 1,174 \\
\hline Brandenburg & 0.04 & $(0.19)$ & 79,055 & 0.04 & $(0.19)$ & 76,437 & $0.04^{\dagger \dagger \dagger}$ & $(0.19)$ & 2,618 & $0.04^{\dagger \dagger \dagger}$ & $(0.21)$ & 1,174 \\
\hline Mecklenburg Western Pomerania & 0.02 & $(0.15)$ & 79,055 & 0.02 & $(0.15)$ & 76,437 & $0.02^{\dagger \dagger \dagger}$ & $(0.15)$ & 2,618 & $0.03^{\text {㸞 }}$ & $(0.16)$ & 1,174 \\
\hline Saxony & 0.06 & $(0.24)$ & 79,055 & 0.06 & $(0.24)$ & 76,437 & $0.06^{\dagger \dagger \dagger}$ & $(0.25)$ & 2,618 & $0.06^{\dagger \dagger \dagger}$ & $(0.24)$ & 1,174 \\
\hline Saxony Anhalt & 0.05 & $(0.21)$ & 79,055 & 0.05 & $(0.22)$ & 76,437 & $0.03^{\dagger \dagger \dagger}$ & $(0.18)$ & 2,618 & $0.03^{\dagger \dagger \dagger}$ & $(0.17)$ & 1,174 \\
\hline Thuringia & 0.05 & $(0.21)$ & 79,055 & 0.05 & $(0.21)$ & 76,437 & $0.04^{\dagger \dagger \dagger}$ & $(0.19)$ & 2,618 & $0.04^{\dagger \dagger \dagger}$ & $(0.19)$ & 1,174 \\
\hline Abroad & 0.00 & $(0.02)$ & 79,055 & 0.00 & $(0.02)$ & 76,437 & $0.00^{\dagger \dagger \dagger}$ & $(0.00)$ & 2,618 & $0.00^{\dagger \dagger \dagger}$ & $(0.00)$ & 1,174 \\
\hline
\end{tabular}

Table to be continued on the next page. 
Table A2 (continued)

\begin{tabular}{|c|c|c|c|c|c|c|c|c|c|c|c|c|}
\hline & \multicolumn{3}{|c|}{ Invited sample } & \multicolumn{3}{|c|}{ Did not sign up } & \multicolumn{3}{|c|}{ Signed up } & \multicolumn{3}{|c|}{ Finale sample } \\
\hline & Mean & (std. dv.) & $\mathrm{N}$ & Mean & & Mean & (std. dv.) & $\mathrm{N}$ & Mean & & Mean & (std. dv.) \\
\hline \multicolumn{13}{|l|}{ Recruitment month (shares) } \\
\hline Pre-test (November, December 2017) & 0.02 & $(0.13)$ & 79,092 & 0.02 & $(0.13)$ & 76,474 & $0.01^{\dagger \dagger \dagger}$ & $(0.09)$ & 2,618 & $0.00^{\dagger \dagger}$ & $(0.07)$ & 1,174 \\
\hline January 2018 & 0.10 & $(0.29)$ & 79,092 & 0.10 & $(0.30)$ & 76,474 & $0.04^{\dagger \dagger \dagger}$ & $(0.19)$ & 2,618 & $0.03^{\dagger \dagger \dagger}$ & $(0.17)$ & 1,174 \\
\hline February 2018 & 0.06 & $(0.24)$ & 79,092 & 0.06 & $(0.25)$ & 76,474 & $0.04^{\dagger \dagger}$ & $(0.19)$ & 2,618 & $0.04^{\dagger \dagger}$ & $(0.19)$ & 1,174 \\
\hline March 2018 & 0.04 & $(0.21)$ & 79,092 & 0.04 & $(0.21)$ & 76,474 & $0.05^{\dagger \dagger \dagger}$ & $(0.22)$ & 2,618 & $0.06^{\dagger \dagger}$ & $(0.23)$ & 1,174 \\
\hline April 2018 & 0.03 & $(0.17)$ & 79,092 & 0.03 & $(0.17)$ & 76,474 & $0.02^{\dagger \dagger}$ & $(0.14)$ & 2,618 & $0.01^{\dagger \dagger}$ & $(0.12)$ & 1,174 \\
\hline May 2018 & 0.05 & $(0.22)$ & 79,092 & 0.05 & $(0.22)$ & 76,474 & $0.05^{\dagger \dagger \dagger}$ & $(0.21)$ & 2,618 & $0.05^{\dagger \dagger}$ & $(0.22)$ & 1,174 \\
\hline June 2018 & 0.05 & $(0.22)$ & 79,092 & 0.05 & $(0.22)$ & 76,474 & $0.06^{\dagger \dagger}$ & $(0.24)$ & 2,618 & $0.06^{\dagger \dagger}$ & $(0.24)$ & 1,174 \\
\hline July 2018 & 0.04 & $(0.20)$ & 79,092 & 0.04 & $(0.20)$ & 76,474 & $0.04^{\dagger \dagger}$ & $(0.19)$ & 2,618 & $0.03^{\dagger \dagger}$ & $(0.18)$ & 1,174 \\
\hline August 2018 & 0.05 & $(0.22)$ & 79,092 & 0.05 & $(0.22)$ & 76,474 & $0.06^{\dagger \dagger}$ & $(0.24)$ & 2,618 & $0.06^{\dagger \dagger \dagger}$ & $(0.24)$ & 1,174 \\
\hline September 2018 & 0.04 & $(0.19)$ & 79,092 & 0.04 & $(0.19)$ & 76,474 & $0.04^{\dagger \dagger}$ & $(0.19)$ & 2,618 & $0.03^{\dagger \dagger}$ & $(0.18)$ & 1,174 \\
\hline October 2018 & 0.04 & $(0.19)$ & 79,092 & 0.04 & $(0.19)$ & 76,474 & $0.03^{\dagger \dagger \dagger}$ & $(0.17)$ & 2,618 & $0.02^{\dagger \dagger \dagger}$ & $(0.15)$ & 1,174 \\
\hline November 2018 & 0.12 & $(0.33)$ & 79,092 & 0.12 & $(0.33)$ & 76,474 & $0.18^{\dagger \dagger \dagger}$ & $(0.39)$ & 2,618 & $0.22^{\dagger \dagger}$ & $(0.42)$ & 1,174 \\
\hline December 2018 & 0.06 & $(0.24)$ & 79,092 & 0.06 & $(0.24)$ & 76,474 & $0.05^{\dagger \dagger}$ & $(0.23)$ & 2,618 & $0.04^{\dagger \dagger}$ & $(0.20)$ & 1,174 \\
\hline January 2019 & 0.07 & $(0.25)$ & 79,092 & 0.07 & $(0.25)$ & 76,474 & $0.06^{\dagger \dagger \dagger}$ & $(0.24)$ & 2,618 & $0.05^{\dagger \dagger}$ & $(0.21)$ & 1,174 \\
\hline February 2019 & 0.06 & $(0.23)$ & 79,092 & 0.06 & $(0.23)$ & 76,474 & $0.05^{\dagger \dagger}$ & $(0.23)$ & 2,618 & $0.05^{\dagger \dagger}$ & $(0.21)$ & 1,174 \\
\hline March 2019 & 0.05 & $(0.21)$ & 79,092 & 0.05 & $(0.21)$ & 76,474 & $0.06^{\dagger \dagger}$ & $(0.23)$ & 2,618 & $0.06^{\dagger \dagger}$ & $(0.23)$ & 1,174 \\
\hline April 2019 & 0.04 & $(0.21)$ & 79,092 & 0.04 & $(0.20)$ & 76,474 & $0.05^{\dagger \dagger \dagger}$ & $(0.21)$ & 2,618 & $0.04^{\dagger \dagger \dagger}$ & $(0.20)$ & 1,174 \\
\hline May 2019 & 0.08 & $(0.26)$ & 79,092 & 0.07 & $(0.26)$ & 76,474 & $0.12^{\dagger \dagger}$ & $(0.32)$ & 2,618 & $0.13^{\dagger \dagger \dagger}$ & $(0.34)$ & 1,174 \\
\hline \multicolumn{13}{|l|}{ Employment status (shares) } \\
\hline Employed, contributing to social insurance & 0.98 & $(0.12)$ & 79,092 & 0.99 & $(0.12)$ & 76,474 & $0.98^{\dagger}$ & $(0.14)$ & 2,618 & 0.98 & $(0.13)$ & 1,174 \\
\hline Marginally employed & 0.00 & $(0.03)$ & 79,092 & 0.00 & $(0.03)$ & 76,474 & $0.00^{\dagger}$ & $(0.00)$ & 2,618 & 0.00 & $(0.00)$ & 1,174 \\
\hline Other (i.e. apprenticeship) & 0.01 & $(0.12)$ & 79,092 & 0.01 & $(0.12)$ & 76,474 & $0.02^{\dagger}$ & $(0.14)$ & 2,618 & 0.02 & $(0.13)$ & 1,174 \\
\hline Part-time job (share) & 0.20 & $(0.40)$ & 77,903 & 0.20 & $(0.40)$ & 75,336 & $0.29^{\dagger \dagger \dagger}$ & $(0.45)$ & 2,567 & $0.34^{\dagger \dagger}$ & $(0.47)$ & 1,153 \\
\hline Daily wage $(5.02-229.05)$ & 97.34 & $(41.51)$ & 77,106 & 96.98 & (41.28) & 74,561 & $107.66^{* * *}$ & $(46.62)$ & 2,545 & $109.30^{* * *}$ & (46.39) & 1,147 \\
\hline Individuals & 79,092 & & & 76,474 & & & 2,618 & & & 1,174 & & \\
\hline
\end{tabular}

Source. Paradata on GJSP participation and IEB version v16. 
Table A3. Figures underlying bias calculation

\begin{tabular}{|c|c|c|c|c|c|c|}
\hline & Invited Sample & $\mathrm{N}$ & Signed Up & $\mathrm{N}$ & Final Sample & $\mathrm{N}$ \\
\hline Female (shares) & 39.99 & 127,198 & 49.81 & 4,698 & 51.09 & 1,873 \\
\hline \multicolumn{7}{|l|}{ Employment status (shares) } \\
\hline $\begin{array}{l}\text { Employed subject to social insurance } \\
\text { contributions }\end{array}$ & 96.77 & 127,201 & 96.72 & 4,698 & 97.49 & 1,873 \\
\hline Marginally employed & 0.15 & 127,201 & 0.15 & 4,698 & 0.11 & 1,873 \\
\hline Other (i.e. apprenticeship) & 3.07 & 127,201 & 3.13 & 4,698 & 2.40 & 1,873 \\
\hline Part-time job (share) & 23.70 & 123,095 & 29.27 & 4,544 & 33.13 & 1,826 \\
\hline \multicolumn{7}{|l|}{ Highest level of qualification (shares) } \\
\hline None & 4.84 & 127,201 & 2.19 & 4,698 & 1.55 & 1,873 \\
\hline Vocational training & 61.07 & 127,201 & 35.21 & 4,698 & 30.38 & 1,873 \\
\hline A-levels & 1.23 & 127,201 & 2.00 & 4,698 & 1.49 & 1,873 \\
\hline A-levels and vocational training & 13.65 & 127,201 & 18.60 & 4,698 & 18.74 & 1,873 \\
\hline Tertiary degree & 19.21 & 127,201 & 42.00 & 4,698 & 47.84 & 1,873 \\
\hline \multicolumn{7}{|l|}{ Age } \\
\hline 17 - 30 years & 22.63 & 127,201 & 28.71 & 4,698 & 26.16 & 1,873 \\
\hline $31-40$ years & 25.70 & 127,201 & 31.76 & 4,698 & 33.64 & 1,873 \\
\hline $41-50$ years & 23.60 & 127,201 & 20.65 & 4,698 & 22.10 & 1,873 \\
\hline $51-60$ years & 28.07 & 127,201 & 18.88 & 4,698 & 18.10 & 1,873 \\
\hline \multicolumn{7}{|l|}{ Daily wage } \\
\hline $5.02-63.53$ euros & 25.00 & 120,266 & 19.45 & 4,412 & 15.49 & 1,795 \\
\hline $63.54-85.44$ euros & 25.00 & 120,266 & 21.31 & 4,412 & 21.00 & 1,795 \\
\hline $85.45-113.71$ euros & 25.00 & 120,266 & 25.25 & 4,412 & 27.24 & 1,795 \\
\hline $113.72-229.35$ euros & 25.01 & 120,266 & 34.00 & 4,412 & 36.27 & 1,795 \\
\hline \multicolumn{7}{|l|}{ Tenure } \\
\hline $0.41-.66$ years & 24.91 & 123,095 & 17.89 & 4,544 & 17.03 & 1,826 \\
\hline $0.66-1.56$ years & 25.08 & 123,095 & 25.81 & 4,544 & 24.04 & 1,826 \\
\hline $1.56-3.86$ years & 25.01 & 123,095 & 30.83 & 4,544 & 32.48 & 1,826 \\
\hline $3.87-22.1$ years & 25.00 & 123,095 & 25.46 & 4,544 & 26.45 & 1,826 \\
\hline \multicolumn{7}{|l|}{ Employment, past 10 years } \\
\hline $0.21-5.43$ years & 25.00 & 127,196 & 32.46 & 4,698 & 32.84 & 1,873 \\
\hline $5.43-7.97$ years & 24.97 & 127,196 & 25.35 & 4,698 & 25.57 & 1,873 \\
\hline 7.97 - 9.6 years & 24.97 & 127,196 & 20.60 & 4,698 & 20.50 & 1,873 \\
\hline $9.6-10$ years & 25.06 & 127,196 & 21.58 & 4,698 & 21.09 & 1,873 \\
\hline Unemployment benefit receipt, past 10 years & 53.74 & 127,196 & 47.30 & 4,698 & 46.61 & 1,873 \\
\hline Welfare benefit receipt, past 10 years & 26.74 & 127,196 & 24.33 & 4,698 & 23.28 & 1,873 \\
\hline Job seeking, past 10 years & 100.00 & 127,196 & 100.00 & 4,698 & 100.00 & 1,873 \\
\hline Participation in policy scheme, past 10 years & 38.03 & 127,196 & 32.03 & 4,698 & 29.47 & 1,873 \\
\hline \multicolumn{7}{|l|}{ Federal state (shares) } \\
\hline Schleswig-Holstein & 3.25 & 127,148 & 2.92 & 4,698 & 2.51 & 1,873 \\
\hline Hamburg & 1.98 & 127,148 & 3.30 & 4,698 & 3.36 & 1,873 \\
\hline Lower Saxony & 10.51 & 127,148 & 9.39 & 4,698 & 10.41 & 1,873 \\
\hline Bremen & 0.93 & 127,148 & 0.96 & 4,698 & 1.23 & 1,873 \\
\hline North Rhine Westphalia & 18.78 & 127,148 & 19.24 & 4,698 & 18.31 & 1,873 \\
\hline Hesse & 6.00 & 127,148 & 5.19 & 4,698 & 5.66 & 1,873 \\
\hline Rhineland Palatinate & 4.50 & 127,148 & 3.60 & 4,698 & 3.36 & 1,873 \\
\hline Baden Württemberg & 9.19 & 127,148 & 10.49 & 4,698 & 10.89 & 1,873 \\
\hline Bavaria & 17.75 & 127,148 & 16.09 & 4,698 & 14.31 & 1,873 \\
\hline Saarland & 1.35 & 127,148 & 1.21 & 4,698 & 1.12 & 1,873 \\
\hline Berlin & 4.70 & 127,148 & 8.98 & 4,698 & 9.29 & 1,873 \\
\hline Brandenburg & 3.71 & 127,148 & 3.53 & 4,698 & 4.16 & 1,873 \\
\hline Mecklenburg Western Pomerania & 2.45 & 127,148 & 2.17 & 4,698 & 2.40 & 1,873 \\
\hline Saxony & 6.22 & 127,148 & 6.30 & 4,698 & 5.98 & 1,873 \\
\hline Saxony Anhalt & 4.39 & 127,148 & 3.30 & 4,698 & 3.47 & 1,873 \\
\hline Thuringia & 4.28 & 127,148 & 3.32 & 4,698 & 3.52 & 1,873 \\
\hline Abroad & 0.02 & 127,148 & 0.00 & 4,698 & 0.00 & 1,873 \\
\hline \multicolumn{7}{|l|}{ Recruitment month (shares) } \\
\hline Pre-test (November, December 2017) & 1.12 & 127,201 & 0.47 & 4,698 & 0.27 & 1,873 \\
\hline January 2018 & 5.97 & 127,201 & 2.09 & 4,698 & 1.98 & 1,873 \\
\hline February 2018 & 3.96 & 127,201 & 2.06 & 4,698 & 2.24 & 1,873 \\
\hline March 2018 & 2.77 & 127,201 & 2.75 & 4,698 & 3.58 & 1,873 \\
\hline April 2018 & 1.90 & 127,201 & 1.19 & 4,698 & 0.91 & 1,873 \\
\hline May 2018 & 3.14 & 127,201 & 2.58 & 4,698 & 3.31 & 1,873 \\
\hline June 2018 & 3.27 & 127,201 & 3.41 & 4,698 & 3.84 & 1,873 \\
\hline July 2018 & 2.52 & 127,201 & 2.02 & 4,698 & 2.19 & 1,873 \\
\hline August 2018 & 6.49 & 127,201 & 7.41 & 4,698 & 7.10 & 1,873 \\
\hline September 2018 & 4.60 & 127,201 & 5.00 & 4,698 & 5.45 & 1,873 \\
\hline October 2018 & 4.50 & 127,201 & 4.30 & 4,698 & 3.36 & 1,873 \\
\hline November 2018 & 15.38 & 127,201 & 19.09 & 4,698 & 22.26 & 1,873 \\
\hline December 2018 & 7.65 & 127,201 & 7.71 & 4,698 & 5.02 & 1,873 \\
\hline January 2019 & 8.58 & 127,201 & 8.07 & 4,698 & 6.09 & 1,873 \\
\hline February 2019 & 7.26 & 127,201 & 6.77 & 4,698 & 5.34 & 1,873 \\
\hline March 2019 & 6.00 & 127,201 & 6.34 & 4,698 & 6.30 & 1,873 \\
\hline April 2019 & 5.48 & 127,201 & 6.05 & 4,698 & 6.09 & 1,873 \\
\hline May 2019 & 9.42 & 127,201 & 12.71 & 4,698 & 14.68 & 1,873 \\
\hline Individuals & 127,201 & & 4,698 & & 1,873 & \\
\hline
\end{tabular}

Source. Paradata on GJSP participation and IEB version $v 16$.

Note. The numbers of observations differ across characteristics as not all characteristics are available for each individual. 
Table A4. Estimated probability of sign up and inclusion in final sample (mass layoffs only)

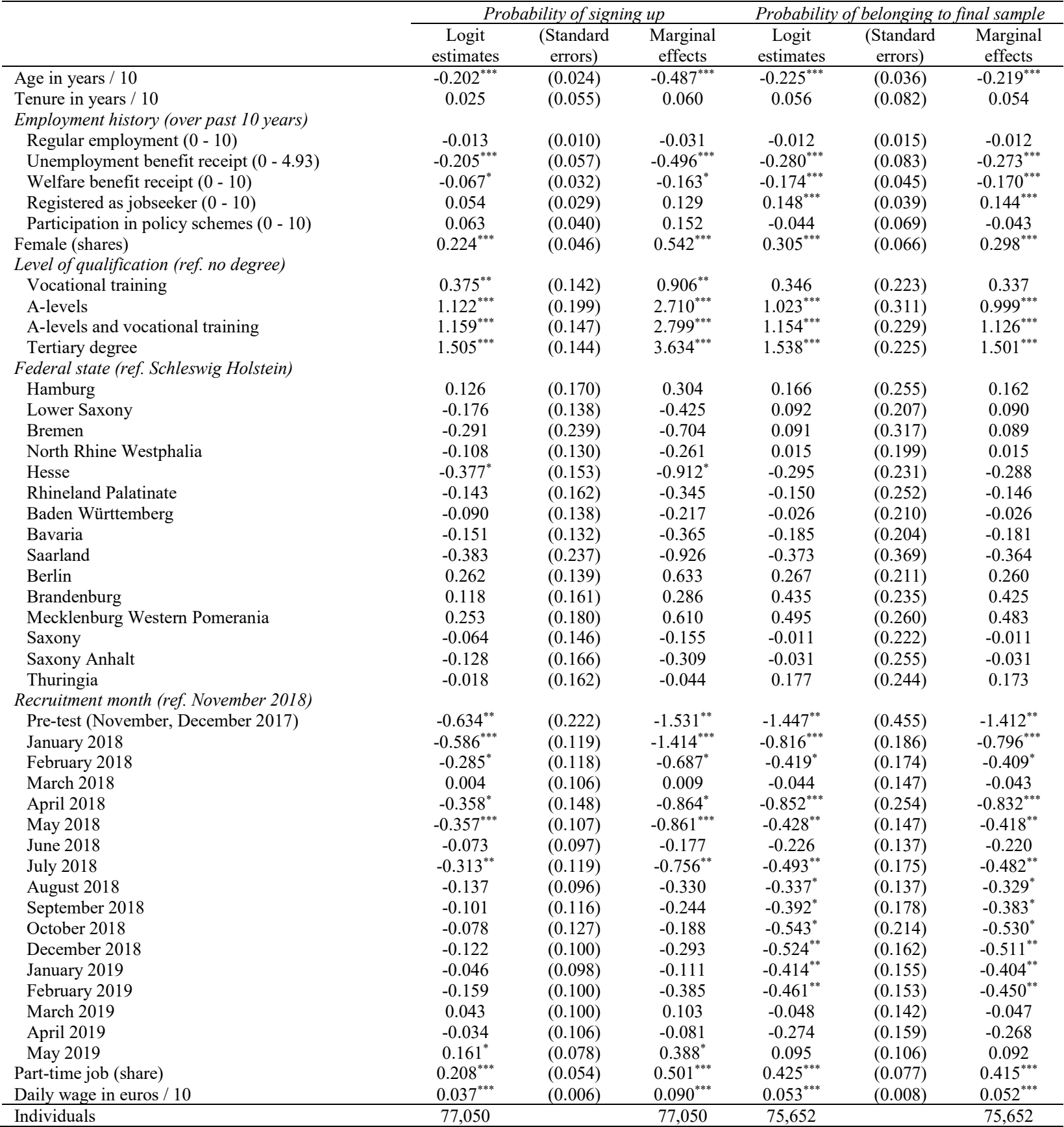

Note. The table presents logit estimates and marginal effects (at means, percentage points) of the probability to sign up / belong to the final sample of the GJSP survey. The number of individuals can be lower compared to Table 2 since only individuals without any missing values on the relevant variables are included in the estimation. ${ }^{* * *} p<0.001,{ }^{* *} p<0.01,{ }^{*} p<0.05$.

Source: Paradata on GJSP participation and IEB version $v 16$. 
Table A5. Consent to record linkage and willingness to participate in cortisol study

\begin{tabular}{|c|c|c|c|c|c|c|c|c|c|c|c|c|}
\hline & \multicolumn{2}{|c|}{ Eligible jobseekers } & \multicolumn{2}{|c|}{ Final sample } & \multicolumn{2}{|c|}{ IEB consent enquired } & \multicolumn{2}{|c|}{ IEB consent given } & \multicolumn{2}{|c|}{ Cortisol enquired } & \multicolumn{2}{|c|}{ Cortisol agreed } \\
\hline & Mean & $N$ & Mean & $N$ & Mean & N & Mean & $N$ & Mean & $N$ & Mean & $N$ \\
\hline Age $(17-60)$ & 41.25 & 127,201 & 38.62 & 1,873 & 38.67 & 1,262 & 38.89 & 1,144 & 38.43 & 1,407 & 38.84 & 1,151 \\
\hline Tenure $(.41-22.1)$ & 3.51 & 123,095 & 3.47 & 1,826 & 3.61 & 1,229 & 3.61 & 1,116 & 3.57 & 1,369 & 3.59 & 1,121 \\
\hline \multicolumn{13}{|l|}{ Employment history (over past 10 years) } \\
\hline Regular employment $(.21-10)$ & 7.27 & 127,196 & 6.79 & 1,873 & 6.82 & 1,262 & 6.87 & 1,144 & 6.83 & 1,407 & 6.86 & 1,151 \\
\hline Unemployment benefit receipt $(0-5.33)$ & 0.48 & 127,196 & 0.33 & 1,873 & 0.32 & 1,262 & 0.31 & 1,144 & 0.31 & 1,407 & 0.32 & 1,151 \\
\hline Welfare benefit receipt $(0-10)$ & 0.71 & 127,196 & 0.47 & 1,873 & 0.46 & 1,262 & 0.46 & 1,144 & 0.45 & 1,407 & 0.45 & 1,151 \\
\hline Registered as jobseeker $(0-10)$ & 1.66 & 127,196 & 1.30 & 1,873 & 1.29 & 1,262 & 1.29 & 1,144 & 1.26 & 1,407 & 1.26 & 1,151 \\
\hline Participation in policy schemes $(0-10)$ & 0.28 & 127,196 & 0.19 & 1,873 & 0.18 & 1,262 & 0.18 & 1,144 & 0.18 & 1,407 & 0.18 & 1,151 \\
\hline Female (shares) & 0.40 & 127,198 & 0.51 & 1,873 & 0.53 & 1,262 & 0.53 & 1,144 & 0.52 & 1,407 & 0.53 & 1,151 \\
\hline Mass layoff sample (share) & 0.62 & 127,201 & 0.63 & 1,873 & 0.62 & 1,262 & 0.62 & 1,144 & 0.63 & 1,407 & 0.62 & 1,151 \\
\hline Academic degree (share) & 0.19 & 127,201 & 0.48 & 1,873 & 0.49 & 1,262 & 0.50 & 1,144 & 0.49 & 1,407 & 0.49 & 1,151 \\
\hline East Germany (share) & 0.21 & 126,654 & 0.20 & 1,867 & 0.20 & 1,257 & 0.21 & 1,141 & 0.20 & 1,402 & 0.21 & 1,147 \\
\hline \multicolumn{13}{|l|}{ Recruitment month (shares) } \\
\hline Pre-test (November, December 2017) & 0.01 & 127,201 & 0.00 & 1,873 & 0.00 & 1,262 & 0.00 & 1,144 & 0.00 & 1,407 & 0.00 & 1,151 \\
\hline January 2018 & 0.06 & 127,201 & 0.02 & 1,873 & 0.01 & 1,262 & 0.01 & 1,144 & 0.02 & 1,407 & 0.02 & 1,151 \\
\hline February 2018 & 0.04 & 127,201 & 0.02 & 1,873 & 0.01 & 1,262 & 0.01 & 1,144 & 0.01 & 1,407 & 0.01 & 1,151 \\
\hline March 2018 & 0.03 & 127,201 & 0.04 & 1,873 & 0.02 & 1,262 & 0.02 & 1,144 & 0.02 & 1,407 & 0.02 & 1,151 \\
\hline April 2018 & 0.02 & 127,201 & 0.01 & 1,873 & 0.01 & 1,262 & 0.01 & 1,144 & 0.01 & 1,407 & 0.01 & 1,151 \\
\hline May 2018 & 0.03 & 127,201 & 0.03 & 1,873 & 0.03 & 1,262 & 0.03 & 1,144 & 0.04 & 1,407 & 0.04 & 1,151 \\
\hline June 2018 & 0.03 & 127,201 & 0.04 & 1,873 & 0.05 & 1,262 & 0.05 & 1,144 & 0.04 & 1,407 & 0.05 & 1,151 \\
\hline July 2018 & 0.03 & 127,201 & 0.02 & 1,873 & 0.02 & 1,262 & 0.02 & 1,144 & 0.02 & 1,407 & 0.02 & 1,151 \\
\hline August 2018 & 0.06 & 127,201 & 0.07 & 1,873 & 0.07 & 1,262 & 0.07 & 1,144 & 0.07 & 1,407 & 0.07 & 1,151 \\
\hline September 2018 & 0.05 & 127,201 & 0.05 & 1,873 & 0.05 & 1,262 & 0.05 & 1,144 & 0.05 & 1,407 & 0.04 & 1,151 \\
\hline October 2018 & 0.04 & 127,201 & 0.03 & 1,873 & 0.03 & 1,262 & 0.03 & 1,144 & 0.03 & 1,407 & 0.03 & 1,151 \\
\hline November 2018 & 0.15 & 127,201 & 0.22 & 1,873 & 0.24 & 1,262 & 0.23 & 1,144 & 0.23 & 1,407 & 0.23 & 1,151 \\
\hline December 2018 & 0.08 & 127,201 & 0.05 & 1,873 & 0.05 & 1,262 & 0.05 & 1,144 & 0.05 & 1,407 & 0.05 & 1,151 \\
\hline January 2019 & 0.09 & 127,201 & 0.06 & 1,873 & 0.06 & 1,262 & 0.06 & 1,144 & 0.06 & 1,407 & 0.06 & 1,151 \\
\hline February 2019 & 0.07 & 127,201 & 0.05 & 1,873 & 0.06 & 1,262 & 0.06 & 1,144 & 0.06 & 1,407 & 0.06 & 1,151 \\
\hline March 2019 & 0.06 & 127,201 & 0.06 & 1,873 & 0.07 & 1,262 & 0.07 & 1,144 & 0.07 & 1,407 & 0.07 & 1,151 \\
\hline April 2019 & 0.05 & 127,201 & 0.06 & 1,873 & 0.06 & 1,262 & 0.06 & 1,144 & 0.06 & 1,407 & 0.06 & 1,151 \\
\hline May 2019 & 0.09 & 127,201 & 0.15 & 1,873 & 0.15 & 1,262 & 0.15 & 1,144 & 0.15 & 1,407 & 0.15 & 1,151 \\
\hline Reward: smartphone (share) & & & 0.10 & 1,873 & 0.10 & 1,262 & 0.10 & 1,144 & 0.09 & 1,407 & 0.10 & 1,151 \\
\hline \multicolumn{13}{|l|}{ Employment status (shares) } \\
\hline Employed, contributing to social insurance & 0.97 & 127,201 & 0.97 & 1,873 & 0.97 & 1,262 & 0.98 & 1,144 & 0.97 & 1,407 & 0.97 & 1,151 \\
\hline Marginally employed & 0.00 & 127,201 & 0.00 & 1,873 & 0.00 & 1,262 & 0.00 & 1,144 & 0.00 & 1,407 & 0.00 & 1,151 \\
\hline Other (i.e. apprenticeship) & 0.03 & 127,201 & 0.02 & 1,873 & 0.03 & 1,262 & 0.02 & 1,144 & 0.03 & 1,407 & 0.03 & 1,151 \\
\hline Part-time job (share) & 0.24 & 123,095 & 0.33 & 1,826 & 0.35 & 1,229 & 0.35 & 1,116 & 0.34 & 1,369 & 0.36 & 1,121 \\
\hline Daily wage $(5.02-229.35)$ & 92.99 & 120,266 & 108.01 & 1,795 & 108.25 & 1,207 & 108.21 & 1,096 & 108.07 & 1,341 & 106.81 & 1,096 \\
\hline Individuals & 127,201 & & 1,873 & & 1,262 & & 1,144 & & 1,407 & & 1,151 & \\
\hline
\end{tabular}

Source. Paradata on GJSP participation and IEB version $v 16$. 
Table A6. Determinants of linkage consent and hair sampling participation (mass layoffs only)

\begin{tabular}{|c|c|c|c|c|c|c|}
\hline & \multicolumn{3}{|c|}{ Probability of Linkage consent } & \multicolumn{3}{|c|}{$\begin{array}{l}\text { Probability of willingness to } \\
\text { participate in the hair sampling }\end{array}$} \\
\hline & $\begin{array}{c}\text { Logit } \\
\text { estimates }\end{array}$ & $\begin{array}{l}\text { (Standard } \\
\text { Errors) }\end{array}$ & $\begin{array}{c}\text { Marginal } \\
\text { effects }\end{array}$ & $\begin{array}{c}\text { Logit } \\
\text { estimates }\end{array}$ & $\begin{array}{l}\text { (Standard } \\
\text { Errors) }\end{array}$ & $\begin{array}{c}\text { Marginal } \\
\text { effects }\end{array}$ \\
\hline Age in years / 10 & 0.319 & $(0.164)$ & $2.295^{*}$ & $0.302^{* *}$ & $(0.116)$ & $4.282^{* *}$ \\
\hline Tenure in years / 10 & -0.620 & $(0.338)$ & -4.465 & -0.182 & $(0.237)$ & -2.589 \\
\hline \multicolumn{7}{|l|}{ Employment history (over past 10 years) } \\
\hline Regular employment $(0-10)$ & 0.124 & $(0.064)$ & $0.893^{*}$ & 0.047 & $(0.045)$ & 0.664 \\
\hline Unemployment benefit receipt $(0-4.93)$ & -0.621 & $(0.417)$ & -4.470 & 0.157 & $(0.295)$ & 2.234 \\
\hline Welfare benefit receipt $(0-10)$ & -0.099 & $(0.203)$ & -0.712 & 0.082 & $(0.148)$ & 1.157 \\
\hline Registered as jobseeker $(0-10)$ & 0.034 & $(0.190)$ & 0.244 & -0.153 & $(0.134)$ & -2.174 \\
\hline Participation in policy schemes $(0-10)$ & 0.630 & $(0.418)$ & 4.535 & 0.043 & $(0.224)$ & 0.612 \\
\hline Female (shares) & -0.290 & $(0.269)$ & -2.090 & 0.271 & $(0.194)$ & 3.842 \\
\hline Academic degree & 0.164 & $(0.316)$ & 1.184 & 0.279 & $(0.223)$ & 3.957 \\
\hline East Germany & $1.316^{* *}$ & $(0.462)$ & $9.477^{* *}$ & 0.338 & $(0.259)$ & 4.802 \\
\hline \multicolumn{7}{|l|}{ Recruitment month (ref. November 2018) } \\
\hline Pre-test (November, December 2017) & 0.000 & (.) & 0.000 & 0.000 & (.) & 0.000 \\
\hline January 2018 & -0.611 & $(0.733)$ & -4.400 & 0.250 & $(0.672)$ & 3.550 \\
\hline February 2018 & 0.678 & $(1.116)$ & 4.878 & 0.011 & $(0.676)$ & 0.149 \\
\hline March 2018 & 0.865 & $(1.074)$ & 6.224 & -0.295 & $(0.513)$ & -4.189 \\
\hline April 2018 & 0.000 & (.) & 0.000 & 0.862 & $(1.066)$ & 12.237 \\
\hline May 2018 & -0.371 & $(0.537)$ & -2.671 & -0.214 & $(0.430)$ & -3.039 \\
\hline June 2018 & 0.603 & $(0.597)$ & 4.342 & -0.006 & $(0.423)$ & -0.084 \\
\hline July 2018 & 0.512 & $(0.800)$ & 3.687 & $-1.069^{*}$ & $(0.455)$ & $-15.162^{*}$ \\
\hline August 2018 & 0.054 & $(0.544)$ & 0.391 & -0.354 & $(0.385)$ & -5.026 \\
\hline September 2018 & 1.108 & $(1.077)$ & 7.980 & -0.377 & $(0.549)$ & -5.350 \\
\hline October 2018 & 0.092 & $(1.100)$ & 0.659 & -0.236 & $(0.690)$ & -3.355 \\
\hline December 2018 & -0.136 & $(0.607)$ & -0.976 & -0.461 & $(0.444)$ & -6.545 \\
\hline January 2019 & -0.005 & $(0.606)$ & -0.033 & -0.778 & $(0.433)$ & -11.035 \\
\hline February 2019 & 0.028 & $(0.589)$ & 0.198 & 0.645 & $(0.564)$ & 9.146 \\
\hline March 2019 & 0.248 & $(0.551)$ & 1.784 & -0.352 & $(0.395)$ & -4.997 \\
\hline April 2019 & 0.105 & $(0.679)$ & 0.759 & 0.304 & $(0.536)$ & 4.310 \\
\hline May 2019 & -0.032 & $(0.412)$ & -0.229 & -0.448 & $(0.300)$ & -6.352 \\
\hline Part-time job (share) & $0.868^{*}$ & $(0.357)$ & $6.246^{*}$ & 0.055 & $(0.250)$ & 0.781 \\
\hline Daily wage in euros / 10 & 0.032 & $(0.040)$ & 0.227 & $-0.063^{*}$ & $(0.027)$ & $-0.900^{*}$ \\
\hline Individuals & 757 & & 757 & 860 & & 860 \\
\hline
\end{tabular}

Note. The table presents logit estimates and marginal effects (at means, percentage points) of the two outcomes (linkage consent, cortisol participation), conditional on being asked to comply with the respective outcome. ${ }^{* * *} p<0.001,{ }^{* *} p<0.01,{ }^{*} p<0.05$.

Source: Paradata on GJSP participation and IEB version $v 16$. 
Table A7. Determinants of continuous participation until waves 7/13 (mass layoffs only)

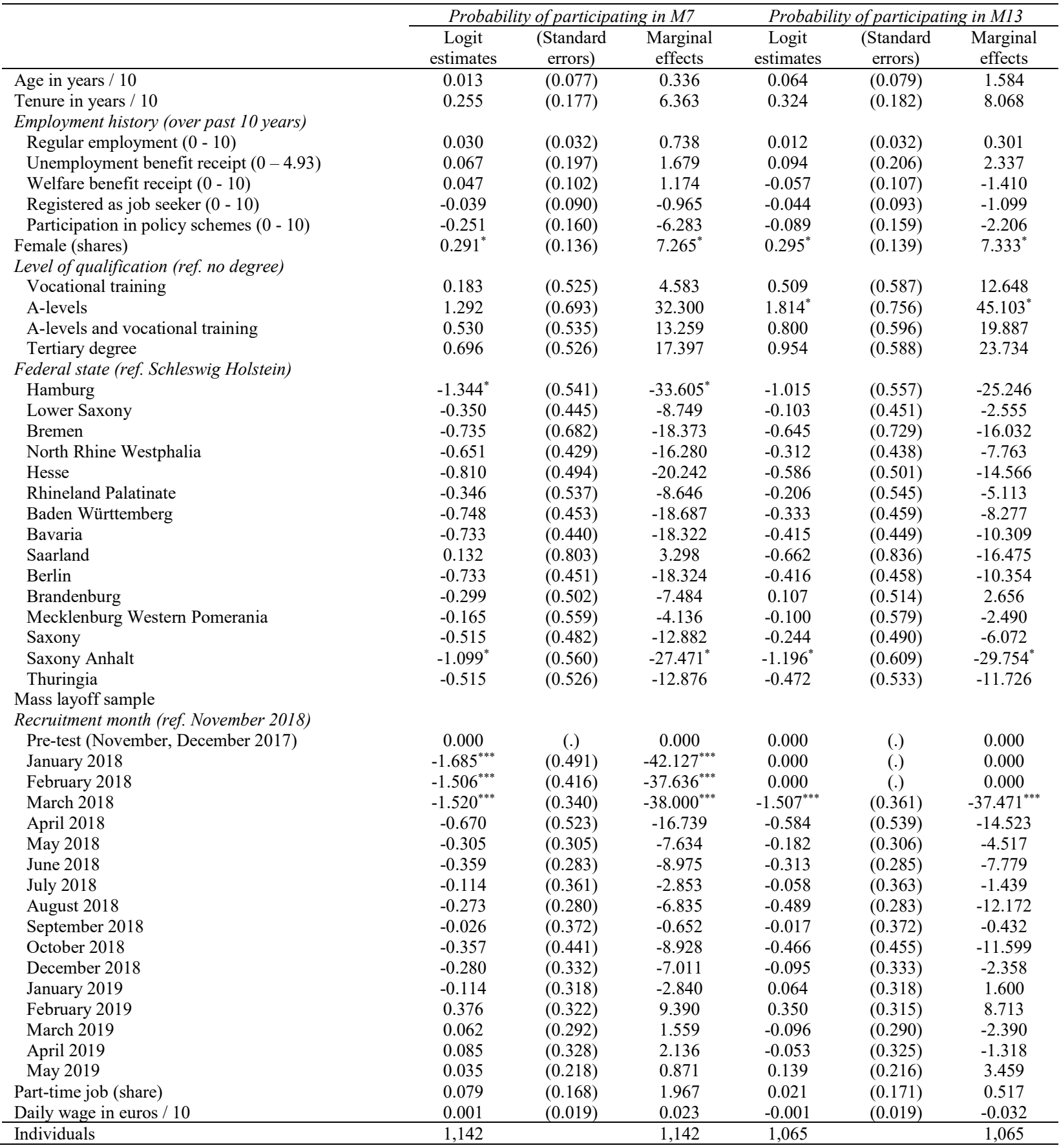

Note. The table presents logit estimates and marginal effects (at means, percentage points) of continued participation after six months and after one year, respectively (conditional on belonging to the final sample). The number of individuals is lower compared to the number of individuals listed in Table A2 since only individuals without any missing values on the relevant variables are included in the estimation. ${ }^{* * *} p<0.001,{ }^{* *} p<0.01,{ }^{*} p<0.05$.

Source: Paradata on GJSP participation and IEB version $v 16$. 


\section{Appendix B: invitation messages}

Material B1: Study Invitation for Letter Condition

\author{
«Mr/Mrs/Ms» «last name» \\ "address» \\ «post code» «city»
}

\author{
Person of Contact \\ Mario Lawes \\ Phone: 030 XXXXXXX \\ Email: $X X X X @ X X X X X . d e$ \\ Nürnberg and Berlin, XX.XX.XXXX
}

Your Experiences matter! Research Project Job Search and Quality of Life!

Dear «Mr/Mrs/Ms» «last name»,

The Freie Universität Berlin and the Institute for Employment Research invite you to take part in our research project "Job Search and Quality of Life". In this study, we investigate the quality of life of people who have recently registered as job seeking at the Federal Employment Agency, but are still employed. With your help, we want to study how changes in your professional career affect quality of life. We want to develop policies that are designed to improve people's well-being!

The key features of the study:

- Your participation is, of course, voluntary. All your answers will be anonymized.

- If you belong to the target group, your participation in the study will take several months und will be rewarded with up to 200 Euro

- To make the participation convenient, the survey will be conducted via a smartphone app.

- For further information, please see the attached leaflet and visit our website www.arbeitssuche-undlebensqualität.de.

Your personal access code to the study: «access code»

With your personal access code, you may register for our research project until «closing date». The survey app is available for download under the search terms Arbeitssuche and Lebensqualität in your AppStore or Playstore, or by scanning the QR codes on the flyer.

In case you do not own a smartphone, we are happy to provide one to you. For requesting a smartphone, you simply register on our project website www.arbeitssuche-und-lebensqualitaet.de.

On the back of this letter, your will find all the relevant information on the protection of your personal data. Your kind support will be greatly appreciated!

With kind regards,

$<<$ Signatures >>

Prof. Dr. Michael Eid, Dr. Clemens Hetschko, Prof. Dr. Ronnie Schöb, Prof. Dr. Gesine Stephan

For further information, please visit our website

www.arbeitssuche-und-lebensqualitaet.de and www.iab.de/de/befragungen.aspx.

Material B2: Data Protection Policy 


\section{Data Protection and Confidentiality}

The Institute for Employment Research of the Federal Employment Agency and the Freie Universität Berlin have launched the research project ,Job Search and Quality of Life'. The survey is conducted by the Happiness Research Organisation. To contact potential participants, the Institute for Employment Research has identified individuals from the data of the Federal Employment Agency who are registered job-seekers.

We hereby certify that any information you provide will be treated with strict confidentiality and will not in any way be retraceable to your person. Your participation in the study is voluntary. Non-participation will not cause you any disadvantages. All the persons involved in the project, at all times, strictly comply with the data protection laws.

How did the Institute for Employment Research obtain your contact details? Was the GDPR (General Data Protection Regulation) complied with?

The Institute for Employment Research is the research institute of the Federal Employment Agency. In accordance with $\$ 282$, section 2 of the Third Book of the German Social Security Code (SGB III), analyzing effects of employment promotion is a key aspect of employment research. Your name and address were retrieved from data supplied by the Federal Employment Agency to the Institute for Employment Research for research purposes. The Institute of Employment Research thereby acted in full compliance with the applicable data protection laws. The fundamental right to data protection is subject to restriction by statue only, which in this case was enacted through $\$ 282$ section 5 of the Social Security Code III, where it reads: „Innerhalb der Bundesagentur dürfen die Daten aus ihrem Geschäftsbereich dem Institut für Arbeitsmarkt- und Berufsforschung zur Verfügung gestellt und dort für dessen Zwecke genutzt und verarbeitet werden." (Within the Federal Employment Agency, all data pertaining to its domain may be made available to the Institute for Employment Research and processed by the latter for research purposes). In the process of adjusting German laws to the GDPR, this particular regulation was not modified, which is in accordance to article 6 section 3 in connection with section 1e) of the GDPR. The Institute for Employment Research is therefore legally entitled to contact any individuals whose contact details are registered at the Federal Employment Agency, without being obliged to ask their prior consent.

\section{What happens to your contact details?}

Any names, addresses and, if applicable, email addresses are handled exclusively by a trustee - the IT department of the Institute for Employment Research - solely for contacting purposes. The Federal Employment Agency / Institute for Employment Research has not and will not disclose any of your contact details to the Freie Universität Berlin or the Happiness Research Organisation. The Freie Universität Berlin is listed on the invitation letter only because it is the inviting institution. It will not have access to any names, addresses or email addresses at any time. Should you decide to participate in the study, the anonymized access code will guarantee the strictly anonymous evaluation of your data. The Happiness Research Organisation will not know your name, address and email address unless you supply it to them yourself. Even in that event, no data will be passed on to any of the researchers involved in the project or to any other parties. Furthermore, the Happiness Research Organisation will delete any data provided to them immediately after the survey has been completed.

\section{What happens to your data?}

Any personal data you provide will be treated strictly confidential and in compliance with applicable data protection regulations. This means that any responses you give will be fully anonymized. There is no way anybody may subsequently learn anything about whether you participated in the study or about your responses.

The Happiness Research Organisation will pass on all data in anonymous form only to the researchers involved at the Institute for Employment Research and the Freie Universität Berlin, or to any other researchers thereafter. You will, of course, be free to leave individual questions unanswered or revoke your participation at any time without giving reasons for it. Upon collection of the data, all the participants' data from the app questioning will be jointly evaluated and published in aggregated form. Any data shared with any third parties will not give any indication as to your person. The data will be used for research purposes only.

The above-listed persons of contact will be glad to answer any further questions you may have on the protection of your data. In addition, please feel free to contact the respective data protection supervisors at the Institute for Employment Research and the Freie Universität Berlin.

Institute for Employment Research: «email address»

Freie Universität Berlin: «email address» 
Material B3: Flyer

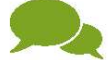

Any further questions?

For detailed information on our research project, the questioning procedure, bonus payments and how to register when you do not have a smartphone, please visit our website: wnww.arbeitssuche-und-lebensqualitaet.de. on the back of the att ached letter, you will find all relevant information on data protection

The Happiness Research Organisation conducts the questioning. For more detailed information regarding the app and how it works, please feel free to contact (0211) $x x x x x x x x$ or $x x x x @ x x x x x x x$.org.

For any other questions, please feel free to contact us Clemens Hets chko: (030) $x x x x x x x$ Mario Lawies:(030) XXXXXXXX $X X X X X X @ X X X X . d e$

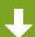

You want to start right away, register and download the app?

We are very happy about that. You will find the app under the search names job search and life quality in your app store or play store or by scanning one of the QR codes:

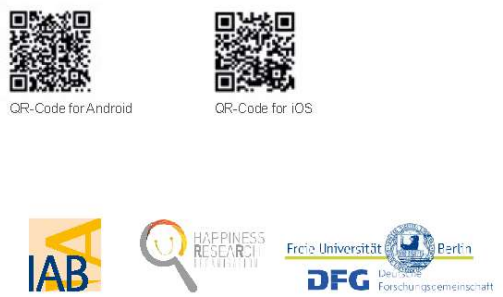

RESEARCH PROJECT JOB SEARCH \& QUALITY OF LIFE

\section{Your Experiences matter!}

Take part in our app-based study and help us find out how job search affects the quality of life.

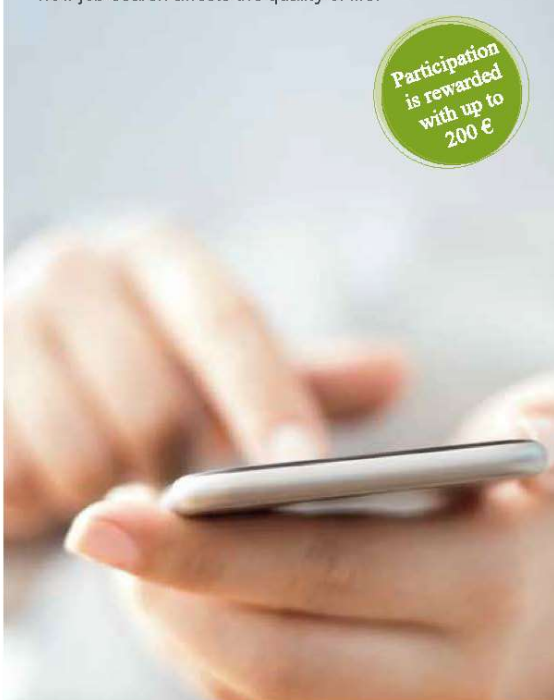

2

How does it work?

To many, working is one of the most important areas of their lives where changes can have significant effects on Ithe quality of ones life. To get a better idea abou the involved processes, we will want to ask you regularly about your welbeing.

But don't worry: Due to the clear and simple design of the questions on the smartphone app, you will easily be able to

In a first round of questions, we clarify if you belong to the target group of the study. After that, the study starts and the app w

\section{两}

Objective stress measure

(optional).

An additional and health-related part of our research project is measuring the stress hormone cortisol. In this part of the study also voluntary, i.e. you may take part in the rest of the stud the study, you simply agree that we may invite you separately to end of the first week of the survey and will provide you with further information.

\section{Or}

What are the next steps?

You may register directly via your smartphone - or online on our website - with your personal access code. You will find your personal access code in the attached letter. 
Material B4: Study Invitation for Email Condition

Dear «Mr/Mrs/Ms» «last name»,

the Freie Universität Berlin and the Institute for Employment Research invite you to take part in our research project. We investigate the quality of life of people who have registered as job-seekers at the German Employment Agency, but are still employed. With your help, we hope to improve the labor market policy in Germany.

The key features of the study:

- Your participation is, of course, voluntary. All your answers will be anonymized.

- If you belong to the target group, your participation in the study will take several months und will be rewarded with up to 200 Euro or a Samsung Galaxy A40.

- To make the participation convenient, the survey will be conducted via a smartphone app.

For more detailed information on the procedure, bonus payments, how to register when you do not have a smartphone, as well as on the protection of your personal data, please visit our website www.arbeitssuche-undlebensqualitaet.de.

Would you like to participate?

Simply download the survey app developed by the Happiness Research Organisation using the search names Arbeitssuche and Lebensqualität from your AppStore or GooglePlayStore. If you are reading this email on your smartphone, you can also just simply click on your type of device iOS or Android. You may register for participation until «closing date» with your personal access code: «access code»

In case you do not own a smartphone and wish to be provided with one, please register using the abovementioned website and your personal access code.

How did we obtain your contact details?

The Institute for Employment Research is a research institute of the Federal Employment Agency, which carries out independent research projects and is legally entitled to use data of the Federal Employment Agency for research purposes, in strict compliance with applicable data protection laws. Detailed information on the protection of your personal data can be found here.

Do you have any further questions?

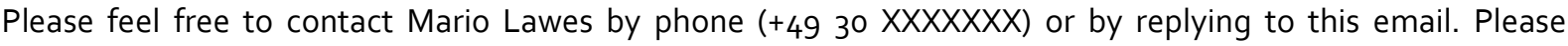
remember to delete your personal access code from your reply email.

Your kind support will be greatly appreciated!

Prof. Dr. Michael Eid, Dr. Clemens Hetschko, Mario Lawes, Prof. Dr. Ronnie Schöb, Prof. Dr. Gesine Stephan

Institute for Employment Research

Research institute of the Federal Employment Agency

Regensburger Straße 100, 90478 Nürnberg

You do not wish to receive any further emails like these?

Please notify us if you do not wish to participate in the research project and do not wish to be contacted by the Institute for Employment Research or its research partners. We will then refrain from sending you any further emails or letters. 
Material B5: Pre-Announcement Letter

\author{
Person of Contact \\ Mario Lawes \\ Phone: $030 X X X X X X X$ \\ Email: $X X X X @ X X X X X . d e$ \\ Nürnberg and Berlin, XX.XX.XXXX
}

\author{
«Mr/Mrs/Ms» «last name» \\ «address» \\ «post code» «city»
}

Your Experiences matter! Pre-Announcement of our app study "Job Search and Quality of Life"!

Dear«Mr/Mrs/Ms» «last name»,

The Freie Universität Berlin and the Institute for Employment Research would like to find out more about the quality of life of people searching for a new job. We, therefore, cordially invite you to take part in our smartphone study "Job Search and Quality of Life". Please help us improve the labor market policies in Germany!

Within the next days, you will receive an email at the email address known to us («email address»). The email will contain a personal access code to register for the study.

If you choose to participate, the survey will go on for several months and will be rewarded with up to 200 Euro or a Samsung Galaxy A40. Please see the attached flyer for more detailed information on the project and the survey procedure.

For the moment, you can just wait for our invitation email. Once you have received your personal access code, you are good to go!

The protection of your personal data is very important to us. On the back of this letter, your will find all the relevant information on data protection and confidentiality.

Your kind support will be greatly appreciated!

$<$ Signatures >>

Prof. Dr. Michael Eid, Dr. Clemens Hetschko, Prof. Dr. Ronnie Schöb, Prof. Dr. Gesine Stephan

For further information, please visit our website

www.arbeitssuche-und-lebensqualitaet.de and www.iab.de/de/befragungen.aspx. 